Article

\title{
The Influence of Single, Tandem, and Clustered DNA Damage on the Electronic Properties of the Double Helix: A Theoretical Study
}

\author{
Bolesław T. Karwowski (D) \\ DNA Damage Laboratory of Food Science Department, Faculty of Pharmacy, Medical University of Lodz, \\ ul. Muszynskiego 1, 90-151 Lodz, Poland; Boleslaw.Karwowski@umed.lodz.pl
}

Received: 13 June 2020; Accepted: 6 July 2020; Published: 8 July 2020

\begin{abstract}
Oxidatively generated damage to DNA frequently appears in the human genome as the effect of aerobic metabolism or as the result of exposure to exogenous oxidizing agents, such as ionization radiation. In this paper. the electronic properties of single, tandem, and clustered DNA damage in comparison with native $d s$-DNA are discussed as a comparative analysis for the first time. A single lesion-8-oxo-7,8-dihydro-2'-deoxyguanosine $\left(\mathrm{G}^{\mathrm{oxo}}\right)$, a tandem lesion- $\left(5^{\prime} S\right)$ and $\left(5^{\prime} R\right)$ $5^{\prime}, 8$-cyclo-2'-deoxyadenosine (cdA), and the presence of both of them in one helix turn as clustered DNA damage were chosen and taken into consideration. The lowest vertical and adiabatic potential (VIP $~ 5.9$ and AIP $\sim 5.5 \mathrm{eV}$, respectively) were found for $\mathrm{G}^{\text {oxo }}$, independently of the discussed DNA lesion type and their distribution within the double helix. Moreover. the VIP and AIP were assigned for $d s$-trimers, $d s$ - dimers and single base pairs isolated from parental $d s$-hexamers in their neutral and cationic forms. The above results were confirmed by the charge and spin density population, which revealed that $\mathrm{G}^{\text {oxo }}$ can be considered as a cation radical point of destination independently of the DNA damage type (single, tandem, or clustered). Additionally. the different influences of cdA on the charge transfer rate were found and discussed in the context of tandem and clustered lesions. Because oligonucleotide lesions are effectively produced as a result of ionization factors. the presented data in this article might be valuable in developing a new scheme of anticancer radiotherapy efficiency.
\end{abstract}

Keywords: DNA damage; electronic properties; charge transfer; DFT; $\left(5^{\prime} R\right) /\left(5^{\prime} S\right)-5^{\prime}, 8-\mathrm{cyclo}-2^{\prime}-$ deoxyadenosine; 8-oxo-7,8-dihydro-2'-deoxyguanosine

\section{Introduction}

DNA is a storage house of genetic information in each cell of a living organism [1]. This information is continuously exposed to different kinds of harmful endo- and exogenous factors, such as ionization radiation (UV, gamma, X-ray), metabolic byproducts, etc. Their interaction with cellular oligonucleotides can cause the formation of DNA lesions both directly and indirectly. Until now, more than 70 types have been identified [2]. It is generally recognized that in the human body, $3 \times 10^{17} \mathrm{DNA}$ damage events per hour take place [3]. On the other hand, DNA lesions can be formed by the activity of the reactive oxygen/nitric species (ROS, RNS) [4]. It has been estimated that approximately $2 \times 10^{4}$ free radical events per cell per day take place. Moreover, their number can increase with physical activity by up to $50 \%$ [5]. Of the plethora of radical oxygen species. the hydroxyl radical $\left({ }^{\bullet} \mathrm{OH}\right)$ has been found as the most reactive, with $k=2-10 \times 10^{-9} \mathrm{M}^{-1} \mathrm{~s}^{-1}$ [6]. From the DNA damage distribution perspective, three main types of lesions can be distinguished: (a) Isolated-one lesion per one helix turn; (b) clustered-two or more per turn; and (c) tandem lesions as a result of a single DNA damage event in which a reactive nucleotide intermediate reacts with an adjacent one [7]. The DNA damage structures discussed in the article are shown in Figure 1. 


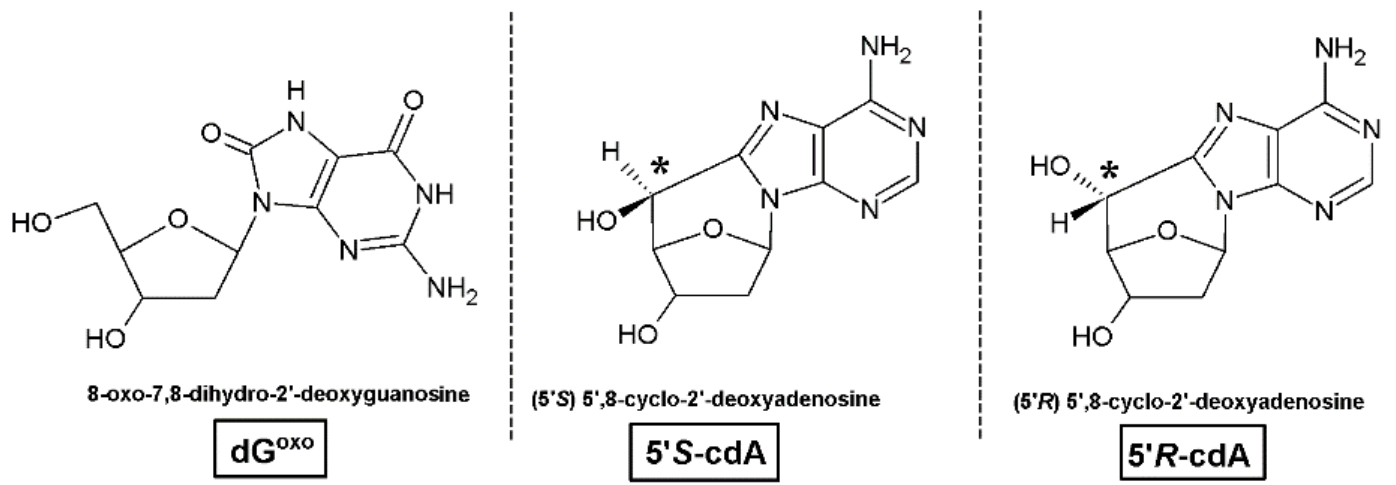

Figure 1. Graphical representation of the structure of the discussed DNA damage.

Among all DNA lesions, 8-oxo-7,8-dihydro-2'-deoxyguanosine $\left(\mathrm{dG}^{\mathrm{oxo}}\right)$ is recognized as the most abundant. Its frequency in a cell has been estimated at $5.5 \times 10^{8}[8,9]$. At the other end of the scale, $\left(5^{\prime} R\right) /\left(5^{\prime} S\right)-5^{\prime}, 8$-cyclo-2'-deoxyadenosine $\left(\left(5^{\prime} R\right)\right.$-cdA and $\left(5^{\prime} S\right)$-cdA) exist, and their frequency in cellular environments has been assigned as unequal $0.07 / 0.93 \times 10^{6} R-c d A / S-c d A$, respectively [10]. However, these results have yet to be verified scientifically and are still under discussion [11,12]. From the cellular point of view, these two diastereomers exhibit different biological/biochemical effects [13-16]. The stability of genetic information is crucial for the future generation of a species, and several repair systems are present in the cell, such as the base/nucleotide repair system (BER, NER), homologous and non-homologous end joining (HEJ, NHEJ), and nucleotide incision repair (NIR) [17]. Their correct activity guarantees the suitable nucleoside sequence in DNA. A failure, however, for example, in NER, can lead to different genetic disorders, cancer, or neurodegenerative disease [18-20].

For all the above repair processes to be effective. the recognition step is the most vital. The cascade of BER proteins starts from the glycosylases' action. These enzymes can recognize and remove simple DNA damage, such as $\mathrm{dG}^{\text {oxo }}, 2^{\prime}$-deoxyuridine [21]. To keep genetic material reproducible and stable, several specific glycosylases exist in cells, for example, OGG1 (8-oxo-guanine glycosylase 1), MutY (adenine DNA glycosylase), and UDG (uracil-DNA glycosylase) [22,23]. On the other hand, due to the additional $\mathrm{C}^{\prime}$ - $\mathrm{C} 8$ covalent bond, neither diastereomer of $\mathrm{cdA}$ is a substrate for the BER system-no $\mathrm{cdA}$-specific glycosylases are known. The tandem lesions in both diastereomeric forms $\left(5^{\prime} R\right)$-cdA and $\left(5^{\prime} S\right)$-cdA are removed from the genome by the more complicated NER machinery. It is important to mention here that these small molecules, depending on the configuration on the $5^{\prime}$ carbon $S$ or $R$, can significantly change the global structure of the DNA double helix, and as a result are removed from the genome at different rates [24]. The structure of the double helix and its changes are commonly described by a DNA standard reference frame. This analysis uses parameters that are useful for hydrogen bonding and base pairs' stacking interaction description, which are fundamental for spatial DNA geometry pronunciation. For further details, please see the work of Olson et al. [25].

From an electronic point of view, $d s$-DNA can be perceived as a conductor of nanofibers [26], which has been shown by Shuster, Barton among others [27,28]. Recently, it was proposed that this phenomenon can allow MutY to scan the genome effectively with the electron transfer mode [29], even though the number of these protein copies is relatively low. MutY is able to verify/scan the E. coli genome $\left(5 \times 10^{5}\right.$ base pairs) within $10 \mathrm{~s}$ [30]. For details, please see the review Barton et al. [31].

In this paper, comparative studies between isolated, clustered, and tandem DNA lesions, contained in $\left(5^{\prime} R\right)-\mathrm{cd} A,\left(5^{\prime} S\right)-\mathrm{cd} A$ and $\mathrm{dG}^{\mathrm{oxo}}$, and their influence on the electronic properties and charge transfer (CT) process of the double helix were considered. It is worth noting that little data exists in the literature that is dedicated to the influence of DNA damage on the hole transfer in ds-DNA [32-34]. 


\section{Results and Discussion}

To elucidate the influence of different types of DNA damage on charge transfer induced by a one-electron oxidizing event, nine double-stranded ( $d s)$ hexamers were chosen (Table 1).

Table 1. Nucleobase sequence "structures" of double-stranded oligonucleotides taken into theoretical consideration. ${ }^{\text {oxo }} \mathrm{G}$ - 8-oxo-7,8-dihydro-2'-deoxyguaosie, (5'S)-cA: $\left(5^{\prime} S\right)$-5 $5^{\prime}, 8$-cyclo-2' -deoxyadenosine, $\left(5^{\prime} R\right)$-cA: $\left(5^{\prime} R\right)-5^{\prime}, 8-$ cyclo-2'-deoxyadenosine.

\begin{tabular}{|c|c|c|}
\hline DNA Damage Type & Oligonucleotide & Oligonucleotide Base Sequence \\
\hline Undamaged Native $d s-\mathrm{DNA}$ & N-DNA & $\mathrm{d}\left[\mathrm{A}_{1} \mathrm{G}_{2} \mathrm{~A}_{3} \mathrm{G}_{4} \mathrm{G}_{5} \mathrm{~A}_{6}\right]^{*} \mathrm{~d}\left[\mathrm{~T}_{6} \mathrm{C}_{5} \mathrm{C}_{4} \mathrm{~A}_{3} \mathrm{C}_{2} \mathrm{~T}_{1}\right]$ \\
\hline \multirow{2}{*}{ Single } & $3 \mathrm{G}^{\mathrm{oxo}}-\mathrm{N}-\mathrm{DNA}$ & $\mathrm{d}\left[\mathrm{A}_{1} \mathrm{G}_{2} \mathrm{~A}_{3}{ }^{\text {oxo }} \mathrm{G}_{4} \mathrm{G}_{5} \mathrm{~A}_{6}\right]^{*} \mathrm{~d}\left[\mathrm{~T}_{6} \mathrm{C}_{5} \mathrm{C}_{4} \mathrm{~A}_{3} \mathrm{C}_{2} \mathrm{~T}_{1}\right]$ \\
\hline & $5 \mathrm{G}^{\mathrm{oxo}}-\mathrm{N}-\mathrm{DNA}$ & $\mathrm{d}\left[\mathrm{A}_{1}{ }^{\mathrm{oxo}} \mathrm{G}_{2} \mathrm{~A}_{3} \mathrm{G}_{4} \mathrm{G}_{5} \mathrm{~A}_{6}\right]^{*} \mathrm{~d}\left[\mathrm{~T}_{6} \mathrm{C}_{5} \mathrm{C}_{4} \mathrm{~A}_{3} \mathrm{C}_{2} \mathrm{~T}_{1}\right]$ \\
\hline Tandem & ScA-DNA & $\mathrm{d}\left[\mathrm{A}_{1} \mathrm{G}_{2}\left(5^{\prime} \mathrm{S}\right) \mathrm{cA}_{3} \mathrm{G}_{4} \mathrm{G}_{5} \mathrm{~A}_{6}\right]^{*} \mathrm{~d}\left[\mathrm{~T}_{6} \mathrm{C}_{5} \mathrm{C}_{4} \mathrm{~A}_{3} \mathrm{C}_{2} \mathrm{~T}_{1}\right]$ \\
\hline \multirow{2}{*}{ Clustered } & $3 \mathrm{G}^{\mathrm{oxo}}-\mathrm{ScA}-\mathrm{DNA}$ & $\mathrm{d}\left[\mathrm{A}_{1} \mathrm{G}_{2}\left(5^{\prime} \mathrm{S}\right) \mathrm{cA}_{3}{ }^{\text {oxo }} \mathrm{G}_{4} \mathrm{G}_{5} \mathrm{~A}_{6}\right]^{*} \mathrm{~d}\left[\mathrm{~T}_{6} \mathrm{C}_{5} \mathrm{C}_{4} \mathrm{~A}_{3} \mathrm{C}_{2} \mathrm{~T}_{1}\right]$ \\
\hline & $5 \mathrm{G}^{\mathrm{oxo}}-\mathrm{ScA}-\mathrm{DNA}$ & $\mathrm{d}\left[\mathrm{A}_{1}{ }^{\text {oxo }} \mathrm{G}_{2}\left(5^{\prime} \mathrm{S}\right) \mathrm{CA}_{3} \mathrm{G}_{4} \mathrm{G}_{5} \mathrm{~A}_{6}\right]^{*} \mathrm{~d}\left[\mathrm{~T}_{6} \mathrm{C}_{5} \mathrm{C}_{4} \mathrm{~A}_{3} \mathrm{C}_{2} \mathrm{~T}_{1}\right]$ \\
\hline Tandem & RcA-DNA & $\mathrm{d}\left[\mathrm{A}_{1} \mathrm{G}_{2}\left(5^{\prime} \mathrm{R}\right) \mathrm{CA}_{3} \mathrm{G}_{4} \mathrm{G}_{5} \mathrm{~A}_{6}\right]^{*} \mathrm{~d}\left[\mathrm{~T}_{6} \mathrm{C}_{5} \mathrm{C}_{4} \mathrm{~A}_{3} \mathrm{C}_{2} \mathrm{~T}_{1}\right]$ \\
\hline \multirow{2}{*}{ Clustered } & 3G ${ }^{\text {oxo-RcA-DNA }}$ & $\mathrm{d}\left[\mathrm{A}_{1} \mathrm{G}_{2}\left(5^{\prime} \mathrm{R}\right) \mathrm{CA}_{3}{ }^{\text {oxo }} \mathrm{G}_{4} \mathrm{G}_{5} \mathrm{~A}_{6}\right]^{*} \mathrm{~d}\left[\mathrm{~T}_{6} \mathrm{C}_{5} \mathrm{C}_{4} \mathrm{~A}_{3} \mathrm{C}_{2} \mathrm{~T}_{1}\right]$ \\
\hline & $5 \mathrm{G}^{\mathrm{oxo}}-\mathrm{RcA}-\mathrm{DNA}$ & $\mathrm{d}\left[\mathrm{A}_{1}{ }^{\mathrm{oxo}} \mathrm{G}_{2}\left(5^{\prime} \mathrm{R}\right) \mathrm{CA}_{3} \mathrm{G}_{4} \mathrm{G}_{5} \mathrm{~A}_{6}\right]^{*} \mathrm{~d}\left[\mathrm{~T}_{6} \mathrm{C}_{5} \mathrm{C}_{4} \mathrm{~A}_{3} \mathrm{C}_{2} \mathrm{~T}_{1}\right]$ \\
\hline
\end{tabular}

The damage of interest was positioned in the central part of ds-oigo. The initial geometry of each in neutral and radical cation forms was optimized at the M062x/D95*:UFF level of theory in the aqueous phase using our own n-layered integrated molecular orbital and molecular mechanics (ONIOM) strategy [35]. The M062x functional was chosen as being suitable for estimating noncovalent interaction as well as for structural studies; additionally. the D95* basis set was used due to its efficiency in calculating such complicated systems in a reasonable time frame [36,37]. The electronic properties of the discussed $d s$-hexamers were obtained at the M062x/6-31+ $\mathrm{G}^{* *}$ level of theory in the aqueous phase. However, due to the nature of DNA solvation. the aqueous phase relaxation influences the vertical ionization potential, and the vertical electron attachment was omitted [38]. This choice was sanctioned by the fact that the double helix is solvated from its outer and not internal shape where the base pair aromatic rings stack. The formed scaffold is the highway for hole migration via the hole hopping or super-exchange mechanism [39]. From the structural point of view, although the optimization of spatial geometry was performed for hexamers, only the central part (tetramer) was given further theoretical consideration. It is well known, and indeed observed in this study too, that the nucleoside pairs located on the $3^{\prime}$ - and $5^{\prime}$-ends of $d s$-DNA adopted a deformed spatial structure on account of the lack of stacking interaction from one of the sides. Their inclusion in the discussion can obscure the clear and correct view of DNA electronic properties as well as charge transfer.

\subsection{Structural Analysis of Isolated, Tandem, and Closured DNA Damage}

The stability of the double helix depends on three factors: The hydrogen bond (HB) energies between complementary bases. the stacking energy within the base pair (BP) dimers, and solvation (first shape water layer) [40]. Although the mutual BP geometry is rather rigid and sensitive to structural changes (for example, crosslink, allylation, loss of bases, or part of the aromatic ring). the global spatial geometry of the double helix is to a greater or lesser extent similar. This phenomenon is derived from the high flexibility of the sugar-phosphate backbone, which keeps bases together in the oligonucleotide strands and prevents them from being scattered. Although the helix spine was taken for geometry optimization, due to its lack of significant meaning for hole transfer and electronic properties, it was removed and is not discussed further. The geometry analysis elucidated that $\mathrm{G}^{\text {oxo }}$ (the isolated lesion) appearing in the investigated double helix structures causes negligible h-rise parameter changes in comparison to the unmodified oligo, independently of its relative position to central $A_{3}$. The h-rise 
parameters are presented in Table 2. The situation is different for the tandem lesion cdAs. These lesions forced h-rise increases, equal for both diastereomers, for the base pair dimers located on the $5^{\prime}$-end and $3^{\prime}$-end direction determined by $\mathrm{cdA}_{3}$. Subsequently, $h$-rise decreases between $\mathrm{cdA}_{3}$ and $\mathrm{G}_{4}$ were observed; however, a higher value was noted for $\left(5^{\prime} S\right)-\mathrm{cd}_{3}$ than $\left(5^{\prime} R\right)-\mathrm{cd} \mathrm{A}_{3}$, which can predict a different influence on the charge transfer process. These observations show that the rigidity of cdA cannot be eliminated by the sugar-phosphate backbone geometry rearmament in comparison to $\mathrm{G}^{\text {oxo }}$.

Table 2. The $h$-rise parameters of base pair dimers obtained for the discussed $d s$-oligonucleotides in their neutral (Neut.) and adiabatic radical cation (ARC) form geometries.

\begin{tabular}{|c|c|c|c|c|c|c|c|c|}
\hline \multicolumn{9}{|c|}{$d s$-DNA Base Dimer $h$-rise Parameter [Å] } \\
\hline \multicolumn{3}{|c|}{ N-DNA } & \multicolumn{3}{|c|}{$3 G^{0 \times 0}$-N-DNA } & \multicolumn{3}{|c|}{$5 G^{0 x 0}-N-D N A$} \\
\hline & NEUT. & ARC & & NEUT. & ARC & & NEUT. & ARC \\
\hline $\mathrm{G}_{2} \mathrm{~A}_{3}$ & 2.96 & 3.01 & $\mathrm{G}_{2} \mathrm{~A}_{3}$ & 3.06 & 3.02 & ${ }^{\text {oxo }} \mathrm{G}_{2} \mathrm{~A}_{3}$ & 2.89 & 2.86 \\
\hline $\mathrm{A}_{3} \mathrm{G}_{4}$ & 3.31 & 2.88 & $\mathrm{~A}_{3} \mathrm{G}_{4}$ oxo & 3.33 & 3.25 & $\mathrm{~A}_{3} \mathrm{G}_{4}$ & 3.24 & 3.23 \\
\hline $\mathrm{G}_{4} \mathrm{G}_{5}$ & 3.34 & 3.14 & ${ }^{\text {oxo }} \mathrm{G}_{4} \mathrm{G}_{5}$ & 3.28 & 3.07 & $\mathrm{G}_{4} \mathrm{G}_{5}$ & 3.34 & 3.38 \\
\hline \multicolumn{3}{|c|}{ ScA-DNA } & \multicolumn{3}{|c|}{$3 G^{\text {oxo }}$-ScA-DNA } & \multicolumn{3}{|c|}{ 5G ${ }^{\text {oxo }}$-ScA-DNA } \\
\hline $\mathrm{G}_{2}\left(5^{\prime} S\right) \mathrm{cA}_{3}$ & 3.36 & 3.29 & $\mathrm{G}_{2}\left(5^{\prime} S\right) \mathrm{cA}_{3}$ & 3.36 & 3.39 & ${ }^{\text {oxo }} \mathrm{G}_{2}\left(5^{\prime} S\right) \mathrm{cA}_{3}$ & 3.26 & 3.11 \\
\hline$\left(5^{\prime} S\right) \mathrm{CA}_{3} \mathrm{G}_{4}$ & 2.98 & 2.82 & $\left(5^{\prime} S\right) \mathrm{CA}_{3} \mathrm{G}_{4}{ }^{\text {oxo }}$ & 3.05 & 2.87 & $\left(5^{\prime} S\right) \mathrm{CA}_{3} \mathrm{G}_{4}$ & 2.94 & 2.97 \\
\hline $\mathrm{G}_{4} \mathrm{G}_{5}$ & 3.68 & 3.56 & ${ }^{\text {oxo }} \mathrm{G}_{4} \mathrm{G}_{5}$ & 3.65 & 3.5 & $\mathrm{G}_{4} \mathrm{G}_{5}$ & 3.68 & 3.68 \\
\hline \multicolumn{3}{|c|}{ RcA-DNA } & \multicolumn{3}{|c|}{ 3G ${ }^{\text {oxo }}$-RcA-DNA } & \multicolumn{3}{|c|}{ 5G Oxo $^{\text {-RcA-DNA }}$} \\
\hline $\mathrm{G}_{2}\left(5^{\prime} R\right) \mathrm{cA}_{3}$ & 3.32 & 3.26 & $\mathrm{G}_{2}\left(5^{\prime} R\right) \mathrm{c} \mathrm{A}_{3}$ & 3.45 & 3.41 & $\begin{array}{c}{ }^{\mathrm{oxo}} \mathrm{G}_{2}\left(5^{\prime} R\right) \mathrm{c} \\
\mathrm{A}_{3}\end{array}$ & 3.37 & 3.24 \\
\hline$\left(5^{\prime} R\right) \mathrm{CA}_{3} \mathrm{G}_{4}$ & 2.8 & 2.7 & $\left(5^{\prime} R\right) \mathrm{CA}_{3} \mathrm{G}_{4}{ }^{\text {oxo }}$ & 3.15 & 3.12 & $\left(5^{\prime} R\right) \mathrm{CA}_{3} \mathrm{G}_{4}$ & 2.87 & 3.05 \\
\hline $\mathrm{G}_{4} \mathrm{G}_{5}$ & 3.6 & 3.49 & ${ }^{\text {oxo }} \mathrm{G}_{4} \mathrm{G}_{5}$ & 3.68 & 3.55 & $\mathrm{G}_{4} \mathrm{G}_{5}$ & 3.63 & 3.67 \\
\hline
\end{tabular}

The second structural parameter that strongly influences the hole migration process is the mutual base pair spatial arrangement. It is worth noting that the aromatic ring overlap (ARO) can be perceived as the outcome of the tilt, twist, slide, shift, and roll standard DNA reference frame parameters. The following differences between native N-DNA and $d s$-oligo containing single, tandem or clustered lesions in the aromatic ring overlapping BP dimers were found (Table 3):

(a) The appearance of $G^{\text {oxo }}$ in the discussed system leads to ARO decreases in all the investigated BP dimers except $\mathrm{G}_{2} \mathrm{~A}_{3}$ of $3 \mathrm{G}^{\text {oxo }}-\mathrm{N}-\mathrm{DNA}$. The above indicates by comparison with $5 \mathrm{G}^{\text {oxo }}$-N-DNA that $\mathrm{G}^{\text {oxo }}$ forces BP flipping from the ideal/parent position in its $3^{\prime}$ - and $5^{\prime}$-end directions.

(b) The tandem lesion appearing in the $d s$-oligonucleotide, i.e., $R$ - or $S$ - cdA, far more strongly disrupts the double helix structure than $G^{\text {oxo }}$. Surprisingly. the presence of $\left(5^{\prime} R\right)$ or $\left(5^{\prime} S\right)$ 5',8-cyclo-2'-deoxyadenosine leads to $\mathrm{G}_{4} \mathrm{G}_{5}$ aromatic ring overlapping increases by $-1.6 \AA^{2}$. On the other hand, $\left(5^{\prime} R\right)$-cdA affected $A R O$ in the case of the $\mathrm{cA}_{3} \mathrm{G}_{4}$ base pair dimer more strongly than $\left(5^{\prime} S\right)$-cdA, with the following values found [in $\AA^{2}$ ]: 0.69 and 1.79 for ScA-DNA and RcA-DNA, respectively. These differences indicate that $\left(5^{\prime} R\right)$-cdA disrupts more strongly the spatial $d s-D N A$ structure than the opposite diastereomer, and therefore the effect of this difference should be visible in the values of the charge transfer process parameters.

(c) The geometrical analysis of the clustered lesion, composed of cdA and $\mathrm{G}^{\text {oxo }}$, reveals that the $\left(5^{\prime} S\right)$-cdA causes ARO increases independently of the relative $\mathrm{G}^{\text {oxo }} 3^{\prime}$ or $5^{\prime}$-end position of cdA. The situation is different in the case of $\left(5^{\prime} R\right)$-cdA if $G^{\text {oxo }}$ is present at the $3^{\prime}$ hydroxyl group site of cdA with a BPs ARO decrease being observed. Contrary to the above, $\mathrm{G}^{\text {oxo }}$ shifted to the $5^{\prime}$ site of $\left(5^{\prime} R\right)$-cdA, causing aromatic ring overlapping increases within the $A_{3} G_{4}$ base pair dimers. Based on the above, it can be expected that in the case of clustered lesions, $\left(5^{\prime} R\right)$-cdA should more strongly affect hole migration than the opposite diastereomer. 
Table 3. Aromatic ring overlapping of the base pair dimers of the discussed $d s$-oligonucleotides in their neutral (Neut.) and adiabatic radical cation (ARC) form geometries.

\begin{tabular}{|c|c|c|c|c|c|c|c|c|}
\hline \multicolumn{9}{|c|}{$d s$-DNA Bases Aromatic Rings Overlap $\left[\AA^{2}\right]$} \\
\hline \multicolumn{3}{|c|}{ N-DNA } & \multicolumn{3}{|c|}{$3 G^{\text {oxo }}$-N-DNA } & \multicolumn{3}{|c|}{$5 G^{0 \times 0}$-N-DNA } \\
\hline & NEUT. & ARC & & NEUT. & ARC & & NEUT. & ARC \\
\hline $\mathrm{G}_{2} \mathrm{~A}_{3}$ & 2.14 & 1.3 & $\mathrm{G}_{2} \mathrm{~A}_{3}$ & 2.95 & 2.9 & $\mathrm{G}_{2}{ }^{\mathrm{oxo}} \mathrm{A}_{3}$ & 1.95 & 1.62 \\
\hline $\mathrm{A}_{3} \mathrm{G}_{4}$ & 3.79 & 3.46 & $\mathrm{~A}_{3} \mathrm{G}_{4}$ oxo & 2.56 & 2.93 & $\mathrm{~A}_{3} \mathrm{G}_{4}$ & 3.31 & 3.66 \\
\hline $\mathrm{G}_{4} \mathrm{G}_{5}$ & 1.28 & 1.07 & $\mathrm{G}_{4}{ }^{\text {oxo }} \mathrm{G}_{5}$ & 0.56 & 0.52 & $\mathrm{G}_{4} \mathrm{G}_{5}$ & 0.77 & 0.83 \\
\hline \multicolumn{3}{|c|}{ ScA-DNA } & \multicolumn{3}{|c|}{$3 G^{\text {oxo }}$-ScA-DNA } & \multicolumn{3}{|c|}{ 5G oxo $_{-}$ScA-DNA } \\
\hline $\mathrm{G}_{2} \mathrm{~A}_{3}$ & 2.29 & 2.2 & $\mathrm{G}_{2} \mathrm{~A}_{3}$ & 2.27 & 2.22 & ${ }^{\text {oxo }} \mathrm{G}_{2} \mathrm{~A}_{3}$ & 1.95 & 2.14 \\
\hline$\left(5^{\prime} S\right) \mathrm{CA}_{3} \mathrm{G}_{4}$ & 3.10 & 5.59 & $\left(5^{\prime} S\right) \mathrm{CA}_{3} \mathrm{G}_{4}{ }^{\text {oxo }}$ & 6.51 & 6.31 & $\left(5^{\prime} S\right) \mathrm{cA}_{3} \mathrm{G}_{4}$ & 5.30 & 5.21 \\
\hline${ }^{\text {oxo }} \mathrm{G}_{4} \mathrm{G}_{5}$ & 2.97 & 3.22 & ${ }^{\text {oxo }} \mathrm{G}_{4} \mathrm{G}_{5}$ & 3.44 & 3.12 & ${ }^{\text {oxo }} \mathrm{G}_{4} \mathrm{G}_{5}$ & 3.40 & 3.42 \\
\hline \multicolumn{3}{|c|}{ RcA-DNA } & \multicolumn{3}{|c|}{ 3G $\mathrm{G}^{\mathrm{oxo}}$-RcA-DNA } & \multicolumn{3}{|c|}{ 5G $\mathrm{G}^{\mathrm{oxo}}$-RcA-DNA } \\
\hline $\mathrm{G}_{2} \mathrm{~A}_{3}$ & 1.99 & 2.11 & $\mathrm{G}_{2} \mathrm{~A}_{3}$ & 0.99 & 0.89 & ${ }^{\text {oxo }} \mathrm{G}_{2} \mathrm{~A}_{3}$ & 2.05 & 1.86 \\
\hline$\left(5^{\prime} R\right) \mathrm{CA}_{3} \mathrm{G}_{4}$ & 2.00 & 5.03 & $\left(5^{\prime} R\right) \mathrm{CA}_{3} \mathrm{G}_{4}$ oxo & 3.27 & 3.13 & $\left(5^{\prime} R\right) \mathrm{CA}_{3} \mathrm{G}_{4}$ & 4.98 & 3.34 \\
\hline${ }^{\text {oxo }} \mathrm{G}_{4} \mathrm{G}_{5}$ & 2.81 & 2.99 & ${ }^{\text {oxo }} \mathrm{G}_{4} \mathrm{G}_{5}$ & 1.86 & 1.99 & ${ }^{\text {oxo }} \mathrm{G}_{4} \mathrm{G}_{5}$ & 2.89 & 0.94 \\
\hline
\end{tabular}

The $h$-rise parameter and ARO are strongly connected with stacking interaction (ST) and have a strong influence on it. This non-covalent interaction is the second force that stabilizes the double helix. The thorough stacking energy analysis (see Table 4) between BP involved directly in the dimer structure shows that the presence of $G^{\text {oxo }}$ leads to stacking energy increases within dimers $A_{2} G_{4}$ and $G_{4} G_{5}$. Surprisingly, when $G^{\text {oxo }}$ is shifted to the $G_{2}$ position, decreases in $S T$ energy of $G_{2} A_{3}$ and $A_{3} G_{4}$ were observed. Similar results were found for all the discussed tandem and clustered lesions, except one, i.e., a stacking interaction energy increase was observed for $G_{2} A_{3}, G_{4} G_{5} B P$ dimers, with a subsequent decrease in the case of $G_{4} G_{5}$. The presence of $G^{\text {oxo }}$ on the $3^{\prime}$-end site of R-cdA (3G $G^{\text {oxo }}$-RcA-DNA) leads to opposite results, with an ST energy decrease noted for the $\mathrm{G}_{2} \mathrm{CA}_{3}$ dimer, while for the remaining two, rises in its value were assigned. The above results strongly indicate that the influence of DNA damage on stacking interaction strongly depends on the oligonucleotide base sequence. However, it should be pointed out that for all the discussed DNA lesions. the ST energy increases in the $\mathrm{G}_{4} \mathrm{G}_{5}$ dimer were observed in a range between 0.04 and $2.79 \mathrm{kcal} / \mathrm{mol}$. These observations indicate that the hole transfer between GG is preferred, which is in good agreement with previous theoretical and experimental data [41].

As mentioned above. the stability of the DNA double helix is the result of stacking and hydrogen bond energies. From previous studies, it is known that base modification and DNA damage can strongly affect mutual complementary base interaction and therefore influence HB energy [34,42]. The results discussed below are presented in Table 5. The comparison of $d s$-DNA containing a single or clustered lesion with native DNA elucidated that the $\mathrm{dG}^{\text {oxo }}$ appearing in the oligonucleotide causes increases in the $\mathrm{HB}$ energy of the $\mathrm{dC}:: \mathrm{dG}^{\text {oxo }}$ pair independently of other lesions present in the range between 0.38 and $0.87 \mathrm{kcal} / \mathrm{mol}$. For a single DNA lesion. the $\mathrm{HB}$ energy increases in the $\mathrm{dC}:: \mathrm{dG}^{\text {oxo }}$ pair were almost the same for $3 \mathrm{G}^{\text {oxo }}-\mathrm{N}-\mathrm{DNA}$ and $5 \mathrm{G}^{\text {oxo }}-\mathrm{N}-\mathrm{DNA}$, i.e., 0.46 and $0.54 \mathrm{kcal} / \mathrm{mol}$, respectively. Moreover, for oligonucleotides containing only $\mathrm{dG}^{\text {oxo }}$. the $\mathrm{HB}$ energies calculated for other base pairs were almost unaffected in comparison with N-DNA, except the $A_{3}:: T_{3}$ base pair of $3 G^{\text {oxo }}-\mathrm{N}-D N A$, for which a fall of $0.28 \mathrm{kcal} / \mathrm{mol}$ was noted. Opposite results were noted for other $d s$-DNA with tandem or clustered lesions in all cases other than for $\mathrm{dC}:: \mathrm{dG}^{\text {oxo }}$ base pairs, where a decrease in $\mathrm{HB}$ energy was observed. The results presented above indicated that clustered or tandem lesions strongly affect the double helix structure and influence its stability. The experimental data shows that $\left(5^{\prime} S\right)$-cdA leads to melting temperature value decreases of $6^{\circ} \mathrm{C}$ [43], while $\mathrm{dG}^{\text {oxo }}$ affected these parameters only negligibly by $2{ }^{\circ} \mathrm{C}$ [44] in comparison to unmodified $d s$-oligo. 
Table 4. Stacking energy interaction in $\mathrm{kcal} / \mathrm{mol}$ within base pairs dimers of the discussed ds-oligonucleotides in their neutral (Neut.) and vertical neutral (after electron adoption by adiabatic radical cation) (VER.N) form geometries.

\begin{tabular}{|c|c|c|c|c|c|c|c|c|}
\hline \multicolumn{9}{|c|}{$d s$-DNA Stacking Energy (kcal/mol) } \\
\hline \multicolumn{3}{|c|}{ N-DNA } & \multicolumn{3}{|c|}{ 3G ${ }^{\text {oxo }}$-N-DNA } & \multicolumn{3}{|c|}{ 5G $\mathrm{G}^{\mathrm{oxo}}$-N-DNA } \\
\hline & NEUT. & VER.N & & NEUT. & VER.N & & NEUT. & VER.N \\
\hline $\mathrm{G}_{2} \mathrm{~A}_{3}$ & -14.56 & -14.55 & $\mathrm{G}_{2} \mathrm{~A}_{3}$ & -14.23 & -14.91 & $\mathrm{G}_{2}{ }^{\text {oxo }} \mathrm{A}_{3}$ & -14.43 & -13.75 \\
\hline $\mathrm{A}_{3} \mathrm{G}_{4}$ & -13.59 & -14.88 & $\mathrm{~A}_{3} \mathrm{G}_{4}$ oxo & -14.69 & -14.63 & $\mathrm{~A}_{3} \mathrm{G}_{4}$ & -13.38 & -14.55 \\
\hline $\mathrm{G}_{4} \mathrm{G}_{5}$ & -12.05 & -13.39 & $\mathrm{G}_{4}{ }^{\mathrm{oxo}} \mathrm{G}_{5}$ & -12.86 & -13.03 & $\mathrm{G}_{4} \mathrm{G}_{5}$ & -12.09 & -12.76 \\
\hline \multicolumn{3}{|c|}{ ScA-DNA } & \multicolumn{3}{|c|}{$3 \mathrm{G}^{\mathrm{oxo}}$-ScA-DNA } & \multicolumn{3}{|c|}{ 5G $\mathrm{G}^{\mathrm{oxo}}$-ScA-DNA } \\
\hline $\mathrm{G}_{2} \mathrm{~A}_{3}$ & -12.94 & -13.10 & $\mathrm{G}_{2} \mathrm{~A}_{3}$ & -12.90 & -13.10 & oxo $\mathrm{G}_{2} \mathrm{~A}_{3}$ & -12.82 & -12.31 \\
\hline$\left(5^{\prime} S\right) \mathrm{CA}_{3} \mathrm{G}_{4}$ & -11.25 & -11.20 & $\left(5^{\prime} S\right) \mathrm{CA}_{3} \mathrm{G}_{4}$ oxo & -11.88 & -11.07 & $\left(5^{\prime} S\right) \mathrm{cA}_{3} \mathrm{G}_{4}$ & -11.40 & -11.90 \\
\hline${ }^{\text {oxo }} \mathrm{G}_{4} \mathrm{G}_{5}$ & -14.15 & -13.27 & ${ }^{\text {oxo }} \mathrm{G}_{4} \mathrm{G}_{5}$ & -14.68 & -13.86 & ${ }^{\text {oxo }} \mathrm{G}_{4} \mathrm{G}_{5}$ & -14.16 & -14.25 \\
\hline \multicolumn{3}{|c|}{ RcA-DNA } & \multicolumn{3}{|c|}{ 3G Gxo $^{\text {-RcA-DNA }}$} & \multicolumn{3}{|c|}{ 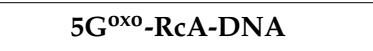 } \\
\hline $\mathrm{G}_{2} \mathrm{~A}_{3}$ & -13.23 & -13.39 & $\mathrm{G}_{2} \mathrm{~A}_{3}$ & -12.92 & -12.86 & ${ }^{\text {oxo }} \mathrm{G}_{2} \mathrm{~A}_{3}$ & -13.05 & -10.58 \\
\hline$\left(5^{\prime} R\right) \mathrm{CA}_{3} \mathrm{G}_{4}$ & -13.39 & -12.52 & $\left(5^{\prime} R\right) \mathrm{CA}_{3} \mathrm{G}_{4}{ }^{\text {oxo }}$ & -14.73 & -13.77 & $\left(5^{\prime} R\right) \mathrm{CA}_{3} \mathrm{G}_{4}$ & -13.24 & -13.70 \\
\hline${ }^{\text {oxo }} \mathrm{G}_{4} \mathrm{G}_{5}$ & -13.50 & -12.92 & ${ }^{\text {oxo }} \mathrm{G}_{4} \mathrm{G}_{5}$ & -14.84 & -14.28 & ${ }^{\text {oxo }} \mathrm{G}_{4} \mathrm{G}_{5}$ & -13.48 & -14.54 \\
\hline
\end{tabular}

Table 5. Hydrogen bond energy in $\mathrm{kcal} / \mathrm{mol}$ of base pairs included in the structure of the discussed $d s$-oligonucleotides in their neutral (Neut.) and vertical neutral (after electron adoption by adiabatic radical cation) (VER.N) form: (a) calculated for an ideal base pair model, (b) calculated for base pairs extracted/selected from 2lsf.pdb [45] and (c) 5iv1.pdb [46] structures.

\begin{tabular}{|c|c|c|c|c|c|c|c|c|}
\hline \multicolumn{9}{|c|}{$d s$-DNA Hydrogen Bond Energy } \\
\hline \multicolumn{3}{|c|}{ N-DNA } & \multicolumn{3}{|c|}{$3 G^{\text {oxo }}$-N-DNA } & \multicolumn{3}{|c|}{$5 \mathrm{G}^{\mathrm{oxo}}$-N-DNA } \\
\hline & NEUT. & VER.N & & NEUT. & VER.N & & NEUT. & VER.N \\
\hline $\mathrm{G}_{2} \mathrm{C}_{2}$ & $\begin{array}{c}-17.23 \\
-17.54^{(a)} \\
-14.36^{(b)}\end{array}$ & $\begin{array}{c}-17.22 \\
-18.10^{(a)}\end{array}$ & $\mathrm{G}_{2} \mathrm{C}_{2}$ & -17.32 & -17.36 & ${ }^{\text {oxo }} \mathrm{G}_{2} \mathrm{C}_{2}$ & -17.69 & -18.16 \\
\hline $\mathrm{A}_{3} \mathrm{~T}_{3}$ & $\begin{array}{c}-10.81 \\
-10.95^{(\mathrm{a})} \\
-8.64^{(\mathrm{b})}\end{array}$ & $\begin{array}{c}-10.74 \\
-9.75 \text { (a) }\end{array}$ & $\mathrm{A}_{3} \mathrm{~T}_{3}$ & -10.53 & -10.44 & $\mathrm{~A}_{3} \mathrm{~T}_{3}$ & -10.80 & -10.39 \\
\hline $\mathrm{G}_{4} \mathrm{C}_{4}$ & -17.20 & -17.00 & ${ }^{\text {oxo }} \mathrm{G}_{4} \mathrm{C}_{4}$ & $\begin{array}{c}-17.74 \\
-18.04^{(a)} \\
-16.83^{(c)}\end{array}$ & $\begin{array}{c}-17.97 \\
-18.49^{(a)}\end{array}$ & $\mathrm{G}_{4} \mathrm{C}_{4}$ & -17.26 & -17.14 \\
\hline $\mathrm{G}_{5} \mathrm{C}_{5}$ & -17.21 & -17.73 & $\mathrm{G}_{5} \mathrm{C}_{5}$ & -17.23 & -17.32 & $\mathrm{G}_{5} \mathrm{C}_{5}$ & -17.23 & -17.31 \\
\hline \multicolumn{3}{|c|}{ ScA-DNA } & \multicolumn{3}{|c|}{$3 G^{\mathbf{0 x o}}$-ScA-DNA } & \multicolumn{3}{|c|}{$5 \mathrm{G}^{\mathrm{oxo}}$-ScA-DNA } \\
\hline $\mathrm{G}_{2} \mathrm{C}_{2}$ & -17.30 & -17.48 & $\mathrm{G}_{2} \mathrm{C}_{2}$ & -17.25 & -17.30 & ${ }^{\text {oxo }} \mathrm{G}_{2} \mathrm{C}_{2}$ & -17.84 & -18.38 \\
\hline$\left(5^{\prime} S\right) \mathrm{CA}_{3} \mathrm{~T}_{3}$ & $\begin{array}{c}-10.62 \\
-10.98^{(a)} \\
-5.89^{(b)}\end{array}$ & $\begin{array}{c}-10.81 \\
-9.77^{(a)}\end{array}$ & $\left(5^{\prime} S\right) \mathrm{A}_{3} \mathrm{~T}_{3}$ & -10.54 & -10.61 & $\left(5^{\prime} S\right) \mathrm{A}_{3} \mathrm{~T}_{3}$ & -10.66 & -9.99 \\
\hline $\mathrm{G}_{4} \mathrm{C}_{4}$ & -17.10 & -17.80 & ${ }^{\mathrm{oxo}} \mathrm{G}_{4} \mathrm{C}_{4}$ & -17.58 & -18.07 & $\mathrm{G}_{4} \mathrm{C}_{4}$ & -17.12 & -17.04 \\
\hline $\mathrm{G}_{5} \mathrm{C}_{5}$ & -17.06 & -17.19 & $\mathrm{G}_{5} \mathrm{C}_{5}$ & -17.08 & -17.23 & $\mathrm{G}_{5} \mathrm{C}_{5}$ & -17.04 & -17.06 \\
\hline \multicolumn{3}{|c|}{ RcA-DNA } & \multicolumn{3}{|c|}{ 3G oxo $^{\text {-RcA-DNA }}$} & \multicolumn{3}{|c|}{ 5G ${ }^{0 x 0}$-RcA-DNA } \\
\hline $\mathrm{G}_{2} \mathrm{C}_{2}$ & -17.19 & -17.15 & $\mathrm{G}_{2} \mathrm{C}_{2}$ & -16.94 & -17.03 & ${ }^{\text {oxo }} \mathrm{G}_{2} \mathrm{C}_{2}$ & -17.81 & -18.01 \\
\hline$\left(5^{\prime} R\right) \mathrm{CA}_{3} \mathrm{~T}_{3}$ & $\begin{array}{c}-10.60 \\
-10.98^{(a)}\end{array}$ & $\begin{array}{c}-10.49 \\
-9.74(\mathrm{a})\end{array}$ & $\left(5^{\prime} R\right) \mathrm{A}_{3} \mathrm{~T}_{3}$ & -10.65 & -10.55 & $\left(5^{\prime} R\right) \mathrm{A}_{3} \mathrm{~T}_{3}$ & -10.65 & -9.88 \\
\hline $\mathrm{G}_{4} \mathrm{C}_{4}$ & -16.77 & -17.86 & ${ }^{\text {oxo }} \mathrm{G}_{4} \mathrm{C}_{4}$ & -18.07 & -18.60 & $\mathrm{G}_{4} \mathrm{C}_{4}$ & -16.82 & -17.50 \\
\hline $\mathrm{G}_{5} \mathrm{C}_{5}$ & -16.95 & -17.17 & $\mathrm{G}_{5} \mathrm{C}_{5}$ & -16.81 & -16.85 & $\mathrm{G}_{5} \mathrm{C}_{5}$ & -16.96 & -16.80 \\
\hline
\end{tabular}




\subsection{The Ionization Potential of Isolated, Tandem, and Closured DNA Damage}

During the genome one-electron oxidation process initiated by, for example, exposure to ionization radiation, radical cations can be formed randomly. The holes (radical cations) can migrate and become trapped at some preferred places of the oligonucleotide structure with the lowest ionization potential. The following order of nucleic base ionization potential (IP) has been noted: thymine $\approx$ cytosine $>$ adenine $>$ guanine; additionally. the following radical distribution has been noted during oligonucleotide $\gamma$-radiation: $35 \% \mathrm{G}^{\bullet+}, 5 \% \mathrm{~A}^{\bullet+}$, and about $45 \%$ of $\mathrm{T}^{\bullet-}$ and $\mathrm{C}^{\bullet-}$ [47]. Therefore, it can be concluded that pyrimidines have a higher ionization potential than purines. Moreover, 8-oxo-2'-deoxyguanine has a lower ionization potential than $\mathrm{dG}$ and is easiest to oxidize [48]. The above results are in good agreement with those presented in this article. Table 6 presents the adiabatic/vertical ionization potential of the isolated base, with base pairs calculated at the M062x/6-31+G** level of theory in the aqueous phase. The situation is a little bit more complicated when $d s$-DAN is taken into consideration. Independently, Senthilkumar and Voityuk calculated the vertical ionization potential (VIP) of all double-stranded tetramers [49,50]. From their study it is clear that the VIP of the tetramers depends on their sequences. In these studies, both the adiabatic and vertical IP of trimers contained within the tetramer structures (extracted as a central part of optimized $d s$-hexamers: Figure 2), as well as $d s$-dimers and isolated base pairs, were taken into theoretical investigation. In all the investigated $d s$-trimers. the lowest VIP and AIP values were found for ( $\left.3 \mathrm{G}^{\text {oxo }}-\mathrm{N}-\mathrm{DNA}\right) \mathrm{A}_{3}{ }^{\text {oxo }} \mathrm{G}_{4} \mathrm{G}_{5}(5,37 / 5,79 \mathrm{eV})$, which are lower by 0.06 and $0.14 \mathrm{eV}$, respectively, than those noted for ${ }^{\text {oxo }} \mathrm{G}_{2} \mathrm{~A}_{3} \mathrm{G}_{4}$. It is important to mention that the lack of oxidized guanosine in the structure eliminates the difference between the vertical and adiabatic state as observed for $\mathrm{A}_{3} \mathrm{G}_{4} \mathrm{G}_{5}$ extracted from $5 \mathrm{G}^{\mathrm{oxo}}-\mathrm{N}-\mathrm{DNA}$. The VIP of this trimer was found at the same level as that of the corresponding one in N-DNA (VIP $=6.02 \mathrm{eV}$ ). As presented in Table 6. the same pattern of VIP and AIP was observed for the discussed tandem and clustered DNA lesions. It is important to mention that the presence of both cdA diastereomers causes slight ionization potential increases in clustered and tandem DNA damage in comparison to the corresponding trimers of native or single-lesioned $d s$-oligo in the following range: VIP: 0.01-0.12 eV and AIP: $0.02-0.05 \mathrm{eV}$. Based on the above, it can be postulated that $\mathrm{G}^{\text {oxo }}$ is a crucial factor, which determines the sink of radical cations and is able to cover the cdA structural influence on the electronic properties of $d s$-trimers.

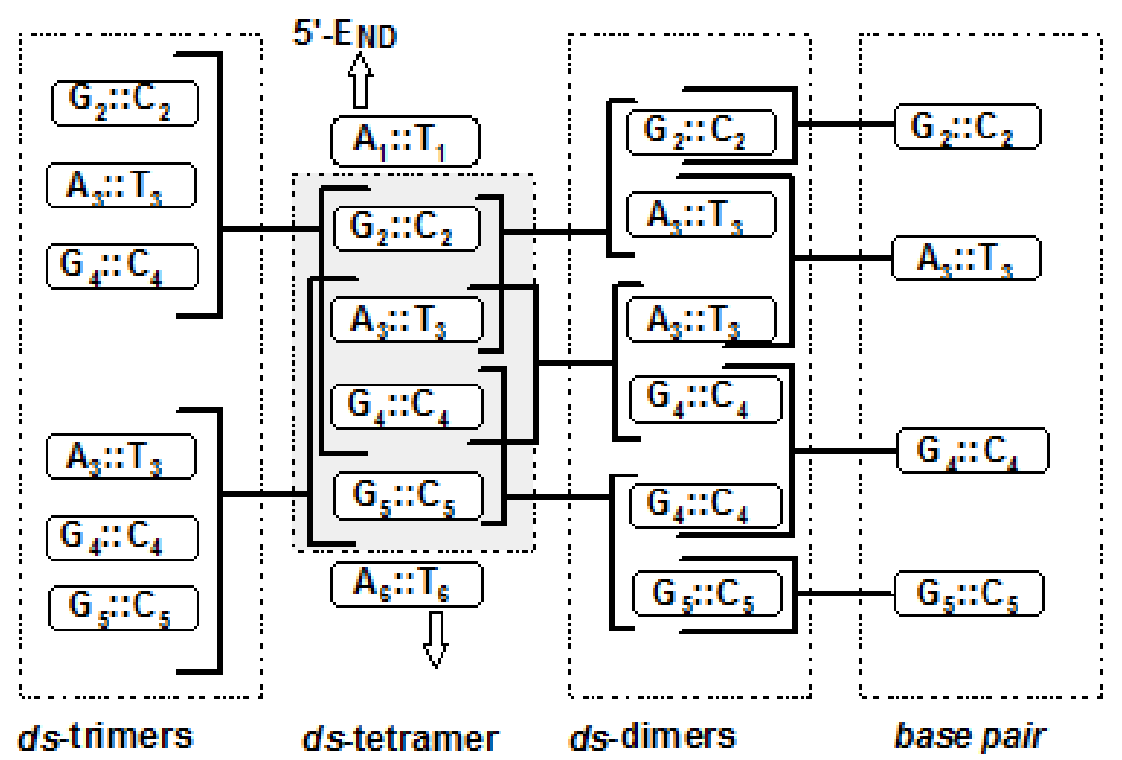

Figure 2. Graphical representation of $d s$-oligonucleotides divided into two $d s$-trimers, three $d s$-dimers, and four base pairs (indicated by dashed squares). 
Table 6. Electronic properties in eV: Vertical (VIP) and adiabatic ionization potential (AIP) of the discussed double-stranded trimers, dimers, as well as single base pairs isolated from their parent $d s$-tetramers, calculated at the M062x/6-31+G** level of theory in the aqueous phase.

\begin{tabular}{|c|c|c|c|c|c|c|c|c|}
\hline & AIP & VIP & & AIP & VIP & & AIP & VIP \\
\hline \multicolumn{9}{|c|}{$d s$-trimers } \\
\hline \multicolumn{3}{|c|}{ N-DNA } & \multicolumn{3}{|c|}{$3 G^{0 x 0}-N-D N A$} & \multicolumn{3}{|c|}{$5 G^{0 x 0}-N-D N A$} \\
\hline $\mathrm{G}_{2} \mathrm{~A}_{3} \mathrm{G}_{4}$ & 5.72 & 6.10 & $\mathrm{G}_{2} \mathrm{~A}_{3}{ }^{\mathbf{0 \times 0}} \mathrm{G}_{4}$ & 5.51 & 5.85 & ${ }^{{ }^{o x}{ }^{\circ}} \mathrm{G}_{2} \mathrm{~A}_{3} \mathrm{G}_{4}$ & 5.45 & 5.90 \\
\hline $\mathrm{A}_{3} \mathrm{G}_{4} \mathrm{G}_{5}$ & 5.64 & 6.03 & $\mathrm{~A}_{3}{ }^{{ }^{\times x o}} \mathrm{G}_{4} \mathrm{G}_{5}$ & 5.37 & 5.79 & $\mathrm{~A}_{3} \mathrm{G}_{4} \mathrm{G}_{5}$ & 6.01 & 6.02 \\
\hline \multicolumn{3}{|c|}{ ScA-DNA } & \multicolumn{3}{|c|}{ 3G ${ }^{\text {oxo }}$-ScA-DNA } & \multicolumn{3}{|c|}{ 5G $\mathrm{G}^{\mathrm{oxo}}$-ScA-DNA } \\
\hline $\mathrm{G}_{2}\left(5^{\prime} S\right) \mathrm{cA}_{3} \mathrm{G}_{4}$ & 5.74 & 6.13 & $\mathrm{G}_{2}\left(5^{\prime} S\right) \mathrm{CA}_{3}{ }^{{ }^{0 \times 0}} \mathrm{G}_{4}$ & 5.51 & 5.91 & ${ }^{{ }^{0 \times 0}} \mathrm{G}_{2}\left(5^{\prime} S\right) \mathrm{cA}_{3} \mathrm{G}_{4}$ & 5.44 & 5.86 \\
\hline$\left(5^{\prime} S\right) \mathrm{cA}_{3} \mathrm{G}_{4} \mathrm{G}_{5}$ & 5.69 & 6.08 & $\left(5^{\prime} S\right) \mathrm{cA}_{3}{ }^{{ }^{0 \times 0}} \mathrm{G}_{4} \mathrm{G}_{5}$ & 5.45 & 5.88 & $\left(5^{\prime} S\right) \mathrm{cA}_{3} \mathrm{G}_{4} \mathrm{G}_{5}$ & 6.09 & 6.14 \\
\hline \multicolumn{3}{|c|}{ RcA-DNA } & \multicolumn{3}{|c|}{$3 G^{\text {oxo }}$-RcA-DNA } & \multicolumn{3}{|c|}{ 5G $\mathrm{G}^{\mathrm{oxo}}$-RcA-DNA } \\
\hline $\mathrm{G}_{2}\left(5^{\prime} R\right) \mathrm{cA}_{3} \mathrm{G}_{4}$ & 5.72 & 6.08 & $\mathrm{G}_{2}\left(5^{\prime} R\right) \mathrm{CA}_{3}{ }^{{ }^{0 \times 0}} \mathrm{G}_{4}$ & 5.50 & 5.94 & ${ }^{{ }^{0 \times 0}} \mathrm{G}_{2}\left(5^{\prime} R\right) \mathrm{cA}_{3} \mathrm{G}_{4}$ & 5.43 & 5.87 \\
\hline$\left(5^{\prime} R\right) \mathrm{CA}_{3} \mathrm{G}_{4} \mathrm{G}_{5}$ & 5.66 & 6.03 & $\left(5^{\prime} R\right) \mathrm{cA}_{3}{ }^{0 \times 0} \mathrm{G}_{4} \mathrm{G}_{5}$ & 5.47 & 5.91 & $\left(5^{\prime} R\right) \mathrm{CA}_{3} \mathrm{G}_{4} \mathrm{G}_{5}$ & 6.13 & 6.03 \\
\hline \multicolumn{9}{|c|}{$d s$-dimers } \\
\hline \multicolumn{3}{|c|}{ N-DNA } & \multicolumn{3}{|c|}{$3 G^{0 \times 0}$-N-DNA } & \multicolumn{3}{|c|}{$5 G^{0 x 0}-N-D N A$} \\
\hline $\mathrm{G}_{2} \mathrm{~A}_{3}$ & 6.13 & 6.15 & $\mathrm{G}_{2} \mathrm{~A}_{3}$ & 6.13 & 6.12 & ${ }^{\text {oxo }} \mathrm{G}_{2} \mathrm{~A}_{3}$ & 5.50 & 5.91 \\
\hline $\mathrm{A}_{3} \mathrm{G}_{4}$ & 5.73 & 6.12 & $\mathrm{~A}_{3}{ }^{\mathrm{oxo}} \mathrm{G}_{4}$ & 5.48 & 5.88 & $\mathrm{~A}_{3} \mathrm{G}_{4}$ & 6.12 & 6.11 \\
\hline $\mathrm{G}_{4} \mathrm{G}_{5}$ & 5.68 & 6.05 & ${ }^{\text {oxo }} \mathrm{G}_{4} \mathrm{G}_{5}$ & 5.40 & 5.83 & $\mathrm{G}_{4} \mathrm{G}_{5}$ & 6.04 & 6.05 \\
\hline \multicolumn{3}{|c|}{ ScA-DNA } & \multicolumn{3}{|c|}{ 3G ${ }^{\text {oxo }}$-ScA-DNA } & \multicolumn{3}{|c|}{$5 G^{\text {oxo }}$-ScA-DNA } \\
\hline $\mathrm{G}_{2} \mathrm{~A}_{3}$ & 6.12 & 6.10 & $\mathrm{G}_{2} \mathrm{~A}_{3}$ & 6.13 & 6.10 & ${ }^{\text {oxo }} \mathrm{G}_{2} \mathrm{~A}_{3}$ & 5.47 & 5.87 \\
\hline$\left(5^{\prime} S\right) \mathrm{cA}_{3} \mathrm{G}_{4}$ & 5.75 & 6.14 & $\left(5^{\prime} S\right) \mathrm{CA}_{3} \mathrm{G}_{4}$ & 5.52 & 5.93 & $\left(5^{\prime} S\right) \mathrm{CA}_{3} \mathrm{G}_{4}$ & 6.20 & 6.15 \\
\hline $\mathrm{G}_{4} \mathrm{G}_{5}$ & 5.78 & 6.12 & $\mathrm{G}_{4} \mathrm{G}_{5}$ & 5.48 & 5.89 & $\mathrm{G}_{4} \mathrm{G}_{5}$ & 6.13 & 6.13 \\
\hline \multicolumn{3}{|c|}{ RcA-DNA } & \multicolumn{3}{|c|}{ 3G oxo $^{\text {-RcA-DNA }}$} & \multicolumn{3}{|c|}{ 5G $\mathrm{G}^{\mathrm{oxo}}$-RcA-DNA } \\
\hline $\mathrm{G}_{2} \mathrm{~A}_{3}$ & 6.12 & 6.10 & $\mathrm{G}_{2} \mathrm{~A}_{3}$ & 6.14 & 6.12 & ${ }^{\text {oxo }} \mathrm{G}_{2} \mathrm{~A}_{3}$ & 5.53 & 5.89 \\
\hline$\left(5^{\prime} R\right) \mathrm{cA}_{3} \mathrm{G}_{4}$ & 5.74 & 6.09 & $\left(5^{\prime} R\right) \mathrm{cA}_{3} \mathrm{G}_{4}$ & 5.51 & 5.94 & $\left(5^{\prime} R\right) \mathrm{cA}_{3} \mathrm{G}_{4}$ & 6.13 & 6.10 \\
\hline $\mathrm{G}_{4} \mathrm{G}_{5}$ & 5.73 & 6.21 & $\mathrm{G}_{4} \mathrm{G}_{5}$ & 5.49 & 5.93 & $\mathrm{G}_{4} \mathrm{G}_{5}$ & 6.21 & 6.21 \\
\hline \multicolumn{9}{|c|}{ Single base pairs } \\
\hline N-D & & & $3 \mathrm{G}^{\mathbf{0 x 0}}-\mathrm{N}-$ & NA & & $5 \mathrm{G}^{0 \times 0}-\mathrm{N}$ & NA & \\
\hline $\mathrm{G}_{2} \mathrm{C}_{2}$ & 6.17 & 6.17 & $\mathrm{G}_{2} \mathrm{C}_{2}$ & 6.19 & 6.19 & ${ }^{\text {oxo }} \mathrm{G}_{2} \mathrm{C}_{2}$ & 5.55 & 5.93 \\
\hline$A_{3} T_{3}$ & 6.63 & 6.65 & $A_{3} T_{3}$ & 6.69 & 6.65 & $A_{3} T_{3}$ & 6.65 & 6.64 \\
\hline $\mathrm{G}_{4} \mathrm{C}_{4}$ & 5.86 & 6.13 & ${ }^{\text {oxo }} \mathrm{G}_{4} \mathrm{C}_{4}$ & 5.55 & 5.91 & $\mathrm{G}_{4} \mathrm{C}_{4}$ & 6.14 & 6.13 \\
\hline $\mathrm{G}_{5} \mathrm{C}_{5}$ & 6.15 & 6.20 & $\mathrm{G}_{5} \mathrm{C}_{5}$ & 6.14 & 6.18 & $\mathrm{G}_{5} \mathrm{C}_{5}$ & 6.19 & 6.20 \\
\hline ScA-1 & & & $3 \mathrm{G}^{\mathrm{oxo}}-\mathrm{ScA}$ & NA & & $5 \mathrm{G}^{\mathrm{oxo}}-\mathrm{Sc} A$ & DNA & \\
\hline $\mathrm{G}_{2} \mathrm{C}_{2}$ & 6.13 & 6.19 & $\mathrm{G}_{2} \mathrm{C}_{2}$ & 6.14 & 6.15 & ${ }^{\text {oxo }} \mathrm{G}_{2} \mathrm{C}_{2}$ & 5.55 & 5.92 \\
\hline$\left(5^{\prime} S\right)_{\mathrm{CA}_{3} \mathrm{~T}_{3}}$ & 6.65 & 6.68 & $\left(5^{\prime} S\right)_{c} A_{3} T_{3}$ & 6.67 & 6.68 & $\left(5^{\prime} S\right) \mathrm{cA}_{3} \mathrm{~T}_{3}$ & 6.83 & 6.69 \\
\hline $\mathrm{G}_{4} \mathrm{C}_{4}$ & 5.84 & 6.14 & ${ }^{\text {oxo }} \mathrm{G}_{4} \mathrm{C}_{4}$ & 5.55 & 5.94 & $\mathrm{G}_{4} \mathrm{C}_{4}$ & 6.19 & 6.18 \\
\hline $\mathrm{G}_{5} \mathrm{C}_{5}$ & 6.19 & 6.22 & $\mathrm{G}_{5} \mathrm{C}_{5}$ & 6.19 & 6.22 & $\mathrm{G}_{5} \mathrm{C}_{5}$ & 6.22 & 6.23 \\
\hline RcA- & & & $3 \mathrm{G}^{\mathrm{oxo}}-\mathrm{RcA}$ & ONA & & $5 \mathrm{G}^{\mathrm{oxo}}-\mathrm{Rc}$ & DNA & \\
\hline $\mathrm{G}_{2} \mathrm{C}_{2}$ & 6.12 & 6.23 & $\mathrm{G}_{2} \mathrm{C}_{2}$ & 6.16 & 6.18 & ${ }^{\text {oxo }} \mathrm{G}_{2} \mathrm{C}_{2}$ & 5.58 & 5.93 \\
\hline$\left.\left(5^{\prime} R\right)\right)_{A_{3}} T_{3}$ & 6.68 & 6.61 & $\left(5^{\prime} R\right) \mathrm{cA}_{3} \mathrm{~T}_{3}$ & 6.65 & 6.61 & $\left(5^{\prime} R\right) \mathrm{cA}_{3} \mathrm{~T}_{3}$ & 6.77 & 6.61 \\
\hline $\mathrm{G}_{4} \mathrm{C}_{4}$ & 5.80 & 6.12 & ${ }^{\text {oxo }} \mathrm{G}_{4} \mathrm{C}_{4}$ & 5.53 & 5.96 & $\mathrm{G}_{4} \mathrm{C}_{4}$ & 6.08 & 6.20 \\
\hline $\mathrm{G}_{5} \mathrm{C}_{5}$ & 6.18 & 6.22 & $\mathrm{G}_{5} \mathrm{C}_{5}$ & 6.15 & 6.19 & $\mathrm{G}_{5} \mathrm{C}_{5}$ & 6.19 & 6.22 \\
\hline & & 1 Base & Pair Model & & & Isolated from $5 \mathrm{iv}$ & pdb [4 & and \\
\hline & & AIP & & VIP & & 21sf.pdb & tructu & \\
\hline G:::C & & 5.58 & & 5.13 & & & & \\
\hline $\mathrm{G}^{\mathrm{oxo}}:: \mathrm{C}$ & & 5.55 & & 5.90 & & $G::: C^{* *}$ & & \\
\hline ScA::T & & 6.35 & & 5.62 & & $\mathrm{G}^{\mathbf{0 x o}}::: \mathrm{C}^{*}$ & & \\
\hline RcA::T & & 6.35 & & 5.62 & & ScA:: $T^{* *}$ & & \\
\hline$A:: T$ & & 6.34 & & 6.62 & & $A:: T^{* *}$ & & \\
\hline
\end{tabular}


The investigated $d s$-tetramers divided into three base pairs $d s$-dimers show that in the structure of native $d s$-DNA. the lowest VIP and AIP were noted for $\mathrm{G}_{4} \mathrm{G}_{5}(6.05 / 5.68 \mathrm{eV})$, which was expected. Only negligible differences between the adiabatic and vertical ionization potential were found for the $G_{2} A_{3}$ moiety. The situation was similar in the case of 3G ${ }^{\text {oxo }}-\mathrm{N}-\mathrm{DNA}$ (single-lesioned ds-oligo), in which the ${ }^{\text {oxo }} \mathrm{G}_{3} \mathrm{G}_{4}$ part becomes a hole trap. The 8-oxo-2'-deoxyguanosine shift into the $\mathrm{G}_{2}$ position changes the pattern of IP distribution. The lowest VIP and AIP was noted for ${ }^{\text {oxo }} \mathrm{G}_{2} \mathrm{~A}_{3} d s$-dimer $(5.91 / 5.50 \mathrm{eV})$, while for other $d s$-dimers, a difference between IPs was not observed. The corresponding results were found for clustered DNA damage in which $A_{3}$ was changed by $\left(5^{\prime} R\right)$ - or $\left(5^{\prime} S\right)$-cdA. It should be pointed out that in all the above-discussed cases. the lowest calculated value of the vertical ionization potential, among the isolated dimers, corresponds to the lowest adiabatic IP. The situation is the opposite in the case of the tandem lesion: A discrepancy between VIP and AIP was noted. The lowest VIP was found for the $\mathrm{G}_{2} \mathrm{cA}_{3}$ ds-dimer of ScA-DNA and the $\mathrm{cA}_{3} \mathrm{G}_{4}$ of RcA-DNA, while the lowest AIP was calculated for $A_{3} G_{4}$ and $G_{4} G_{5}$, respectively (Table 6). These observations indicate that cdA appearing in the double helix leads to structural changes, which can obscure the charge migration process. As focused on in Table 3. the geometry rearrangement and its energetical pronunciation (Tables 4 and 5) are more visible after one-electron oxidation. To confirm the above results, parent $d s$-tetramers were divided into four single base pairs for which the VIP and AIP were calculated at the M062x/6-31+G** level of theory in the aqueous phase. The obtained results show that in the case of $\mathrm{dG}^{\text {oxo }}$ being absent. the lowest VIP and AIP were assigned for $\mathrm{G}_{4} \mathrm{C}_{4}$ BP independently of which ds-tetramer (native, single, tandem, or cluster lesioned) was isolated. As can be expected for the rest of the discussed ds-oligos. the lowest value of vertical and adiabatic IP was found for base pairs that contained $G^{\text {oxo }}$ in their moiety. Moreover, almost the same values of these parameters were noted for $\mathrm{G}_{4} \mathrm{C}_{4}$ and ${ }^{\text {oxo }} \mathrm{G}_{3} \mathrm{C}_{3}: 6.1 / 5.8 \mathrm{eV}$ and 5.9/5.5 eV VIP /AIP, respectively. These results are in good agreement with the experimental data, which shows that the $5^{\prime}$-end GC pair in the $\mathrm{d}[\mathrm{GG}]^{*} \mathrm{~d}[\mathrm{CC}]$ dimer is the most easily oxidized (due to it having the lowest VIP and AIP) [51]. Additionally, in each discussed case, ${ }^{\text {oxo }}$ GC BP had a lower VIP and AIP by $0.3 \mathrm{eV}$ than the parent GC pair as shown in Table 6. For the remaining base pairs. the assigned IP values fluctuated. However, what is surprising is that the VIP was mainly noted as lower or at the same level as the adiabatic IP. Based on an ionization potential and structural analysis, it can be concluded that the hole migrated through $d s$-DNA without each BP structural rearrangement, which is necessary for the VIP $\rightarrow$ AIP conversion. Therefore. the hole slides through the double helix until it settles in the "pleasant" part of $d s$-oligo, thanks to it having the lowest VIP and AIP.

A comparative spatial geometry analysis of the discussed $d s$-tetramer between their initial geometry of adiabatic neutral and positively charged states shows that native N-DNA and 5G ${ }^{\text {oxo-RcA-DNA are }}$ the most sensitive to adiabatic radical cation formation (Table 7). In other cases. the presence of $d_{G}{ }^{\text {oxo }}$ or cdA eliminate the structural changes forced by electron loss of $d s$-oligo. It can be predicted that DNA damage formation makes the hole transfer process much easier towards the radical cation sink formed by $\mathrm{dG}^{\text {oxo }}$ than in the case of unmodified $d s$-oligo, which required significant double helix changes for positive charge compensation. The rigidity of $\left(5^{\prime} R / 5^{\prime} S\right)$-cdA (tandem lesion) makes the $d s$-DNA structure resistant to positive ionization. Additionally. the appearance of clustered damage formed by cdA and $\mathrm{dG}^{\text {oxo }}$ in the case of $5 \mathrm{G}^{\text {oxo }}$-RcA-DNA leads to significant geometry changes (Table 7 ) in comparison to others.

In the ionization potential analysis presented above, differences between the discussed $d s$-oligo were observed forcing the comprehensive charge and spin analysis, presented in Table 8. As expected, independently of the type of $d s$-oligo, whether undamaged, isolated, tandem, or clustered lesioned. the charge and spin are mainly located on $\mathrm{G}^{\text {oxo }}$ or the $5^{\prime} \mathrm{G}_{4}$ of the $\mathrm{G}_{4} \mathrm{G}_{5}$ dimer in each case. Moreover. the difference between the vertical and adiabatic radical cation form of $d s$-DNA was negligible. These observations confirm the results (ionization potential) discussed above, which indicate that independently of the system after a complete dismantle of $d s$-DNAs into constituent base pairs, G ${ }^{\text {oxo }}$ or $5^{\prime} \mathrm{G}_{4}$ of $\mathrm{G}_{4} \mathrm{G}_{5}$ can be considered a suitable part of the double helix for positive charge accumulation. 
Table 7. RMSD (root-mean-square deviation) in $\AA$ of the atomic positions calculated for $d s$-DNAs in neutral and cation radical forms.

\begin{tabular}{cccc}
\hline$d s-o l i g o$ & Backbone & Bases & All Nucleic Acid \\
\hline N-DNA & 1.347 & 1.039 & 1.197 \\
\hline 3G ${ }^{\text {oxo }}$-N-DNA & 0.299 & 0.225 & 0.261 \\
\hline 5G ${ }^{\text {oxo }}$-N-DNA & 0.359 & 0.319 & 0.337 \\
\hline ScA-DNA & 0.699 & 0.193 & 0.516 \\
\hline 3G ${ }^{\text {oxo }}-S c A-D N A$ & 0.203 & 0.176 & 0.190 \\
\hline 5G ${ }^{\text {oxo-ScA-DNA }}$ & 0.247 & 0.214 & 0.231 \\
\hline RcA-DNA & 0.695 & 0.298 & 0.537 \\
\hline 3G ${ }^{\text {oxo }}-$ RcA-DNA & 0.119 & 0.082 & 0.103 \\
\hline 5G ${ }^{\text {oxo }}-$ RcA-DNA & 1.032 & 0.817 & 0.931 \\
\hline
\end{tabular}

As mentioned above. the charge transfer through the double helix independently of the damage type can be described as a super-exchange or multistep hopping process [28,34]. Using the previously described strategy. the barrier $(\Delta G)$ for hole transfer within interlaced trimers was assigned in vertical and adiabatic modes (Figure 2, Table 9) [42]. It was found that in all the discussed $d s$-oligonucleotides. the "hole" appearing in the double-helix structure preferably migrated to $G_{4}$ or $G^{\text {oxo }}$ independently of its position in the $d s$-DNA. These results are in excellent agreement with the experimental data obtained by Schuster [41].

The charge transfer migration through the double helix can be described according to the Marcus theory, which states that the rate constant $\left(k_{\mathrm{ET}}\right)$ of charge transfer $(\mathrm{CT})$ depends on several factors: The structure of $\pi$-stacks, i.e., BPs. the driving force $(\Delta F)$, nuclear reorganization $(\lambda)$, activation $\left(E_{\mathrm{a}}\right)$ and the electron-coupling $\left(V_{12}\right)$ energies [52]. $V_{12}$ was calculated according to the GMH (generalized Mulliken-Hush) strategy within the terms of the occupied Kohn-Sham orbital method [53,54]. The charge transfer, which passes through the adiabatic states of the donor and acceptor, is associated with the movement of internal geometries (atoms), expressed by $\lambda$ in the Marcus theory. All the above parameters calculated for the systems discussed in this article are presented in Table 10.

An analysis of the reorganization energies reveals a significant rise in the $A_{3} G_{4}, G_{4} G_{5}$ dimer in the case of native unmodified N-DAN and for $\mathrm{cdA}_{3} \mathrm{G}_{4}, \mathrm{G} 4 \mathrm{G} 4$ of $d s$-DNA containing a tandem lesion. Moreover. the same was noted when $\mathrm{G}_{4}$ was converted into $\mathrm{G}^{\text {oxo }}$ ( $3 \mathrm{G}^{\text {oxo }}-\mathrm{N}-\mathrm{DNA}$, 3G ${ }^{\text {oxo }}-\mathrm{RcA}-\mathrm{DNA}$, $3 \mathrm{G}^{\text {oxo }}$-ScA-DNA). It is important to mention that in the case of damage being present in the double helix. the reorganization energy of the $A_{3} G_{4}$ dimer is almost equal to that found for $G_{4} G_{5}$, while for unmodified ds-oligo (N-DNA). the $\lambda$ of $G_{4} G_{5}$ was two times higher than for $A_{3} G_{4}$. For the oligonucleotides where $G^{\text {oxo }}$ changed to the $G_{2}$ position. the highest $\lambda$ was denoted for the $G_{2}{ }^{\text {oxo }} A_{3}$ dimer (approximately $0.40 \mathrm{eV}$ ) while for the remaining dimers. the value was significantly lower (0.01-0.04 eV). However, for 5G $G^{\text {oxo }}-$ RcA-DNA. the $\lambda$ of $A_{3} G_{4}$ and $G_{4} G_{5}$ should be noted as follows: 0.16 and $0.12 \mathrm{eV}$ respectively. This strongly indicates that $\mathrm{G}^{\text {oxo }}$ plays an invaluable role in genome protection, taking the role of radical slope/trash instead of both diastereomers of cdA (tandem lesion). Due to the fact that $k_{\mathrm{HT}}$ is strongly dependent on the distance and aromatic ring overlapping between the donor and acceptor, an influence of the single, tandem, and clustered DNA lesion on charge transfer in the double helix shape can be expected in comparison with unmodified $d s-D A N$. Table 9 presents the discussed parameters of the charge transfer process. 
Table 8. Hirshfeld charge (Q) and spin (S) in [au] distribution in the shape of ds-oligonucleotides only nucleosides bases were taken into consideration, calculated at the M062X/D95*//M062x/6-31+G** level of theory in the aqueous phase. A—neutral, VC—vertical cation, C—adiabatic cation.

\begin{tabular}{|c|c|c|c|c|c|c|c|c|c|c|c|c|c|c|c|c|c|}
\hline \multicolumn{18}{|c|}{$d s$-DNA Hirshfeld Charge and Spin Density Population } \\
\hline \multicolumn{6}{|c|}{ N-DNA } & \multicolumn{6}{|c|}{$3 \mathrm{G}^{\mathrm{oxo}}$-N-DNA } & \multicolumn{6}{|c|}{$5 G^{\text {oxo }}-\mathrm{N}-\mathrm{DNA}$} \\
\hline & $\mathbf{N}$ & \multicolumn{2}{|c|}{ VC } & \multicolumn{2}{|c|}{ C } & & $\mathbf{N}$ & \multicolumn{2}{|c|}{ VC } & \multicolumn{2}{|c|}{ C } & & $\mathbf{N}$ & \multicolumn{2}{|c|}{ VC } & \multicolumn{2}{|c|}{ C } \\
\hline & $\mathbf{Q}$ & $\mathbf{Q}$ & S & $\mathbf{Q}$ & $\mathbf{S}$ & & $\mathbf{Q}$ & $\mathbf{Q}$ & $\mathbf{S}$ & $\mathbf{Q}$ & $\mathbf{S}$ & & $\mathbf{Q}$ & Q & $\mathbf{S}$ & Q & $\mathrm{S}$ \\
\hline $\mathrm{T}_{6}$ & -0.05 & -0.05 & & -0.04 & & $T_{6}$ & -0.05 & -0.05 & & -0.04 & & $T_{6}$ & -0.05 & -0.05 & & -0.05 & \\
\hline $\mathrm{C}_{5}$ & 0.18 & 0.19 & & 0.18 & & $\mathrm{C}_{5}$ & 0.17 & 0.17 & & 0.17 & & $\mathrm{C}_{5}$ & 0.18 & 0.18 & & 0.17 & \\
\hline $\mathrm{C}_{4}$ & 0.19 & 0.22 & & 0.32 & & $\mathrm{C}_{4}$ & 0.21 & 0.24 & & 0.29 & & $\mathrm{C}_{4}$ & 0.19 & 0.19 & & 0.20 & \\
\hline$T_{3}$ & -0.07 & -0.05 & & -0.02 & & $T_{3}$ & -0.07 & -0.05 & & -0.05 & & $T_{3}$ & -0.06 & -0.06 & & -0.05 & \\
\hline $\mathrm{C}_{2}$ & 0.21 & 0.22 & & 0.22 & & $\mathrm{C}_{2}$ & 0.20 & 0.21 & & 0.21 & & $\mathrm{C}_{2}$ & 0.21 & 0.25 & & 0.31 & \\
\hline$T_{1}$ & -0.08 & -0.07 & & -0.07 & & $T_{1}$ & -0.08 & -0.07 & & -0.07 & & $T_{1}$ & -0.08 & -0.05 & & -0.02 & \\
\hline $\mathbf{A}_{1}$ & 0.01 & 0.01 & & 0.00 & & $\mathbf{A}_{1}$ & 0.01 & 0.01 & & 0.01 & & $\mathbf{A}_{1}$ & 0.02 & 0.06 & 0.01 & 0.05 & 0.02 \\
\hline $\mathrm{G}_{2}$ & -0.14 & -0.13 & & -0.14 & & $\mathrm{G}_{2}$ & -0.14 & -0.13 & & -0.13 & & $\mathrm{G}_{2}{ }^{\mathrm{oxo}}$ & -0.16 & 0.69 & 0.97 & 0.59 & 0.97 \\
\hline $\mathbf{A}_{3}$ & 0.03 & 0.06 & 0.02 & 0.05 & 0.03 & $\mathbf{A}_{3}$ & 0.03 & 0.07 & 0.02 & 0.07 & 0.02 & $\mathbf{A}_{3}$ & 0.03 & 0.06 & 0.02 & 0.05 & 0.01 \\
\hline $\mathrm{G}_{4}$ & -0.18 & 0.67 & 0.96 & 0.55 & 0.96 & $\mathrm{G}_{4}{ }^{\text {oxo }}$ & -0.19 & 0.66 & 0.97 & 0.59 & 0.97 & $\mathrm{G}_{4}$ & -0.18 & -0.17 & & -0.16 & \\
\hline $\mathrm{G}_{5}$ & -0.14 & -0.10 & 0.02 & -0.09 & 0.02 & $\mathrm{G}_{5}$ & -0.13 & -0.10 & 0.01 & -0.10 & 0.01 & $\mathrm{G}_{5}$ & -0.14 & -0.14 & & -0.13 & \\
\hline $\mathbf{A}_{6}$ & 0.03 & 0.04 & & 0.03 & & $A_{6}$ & 0.04 & 0.04 & & 0.05 & & $A_{6}$ & 0.04 & 0.04 & & 0.04 & \\
\hline \multicolumn{6}{|c|}{ ScA-DNA } & \multicolumn{6}{|c|}{$3 G^{0 \times 0}$-ScA-DNA } & \multicolumn{6}{|c|}{ 5G $\mathrm{G}^{\mathrm{oxo}}$-ScA-DNA } \\
\hline $\mathrm{T}_{6}$ & -0.06 & -0.06 & & -0.05 & & $T_{6}$ & -0.06 & -0.06 & & -0.05 & & $T_{6}$ & -0.06 & -0.06 & & -0.06 & \\
\hline $\mathrm{C}_{5}$ & 0.21 & 0.22 & & 0.22 & & $\mathrm{C}_{5}$ & 0.21 & 0.22 & & 0.22 & & $\mathrm{C}_{5}$ & 0.21 & 0.21 & & 0.22 & \\
\hline $\mathrm{C}_{4}$ & 0.13 & 0.16 & & 0.23 & & $\mathrm{C}_{4}$ & 0.13 & 0.16 & & 0.23 & & $\mathrm{C}_{4}$ & 0.12 & 0.12 & & 0.13 & \\
\hline $\mathrm{T}_{3}$ & -0.08 & -0.07 & & -0.06 & & $T_{3}$ & -0.08 & -0.07 & & -0.07 & & $T_{3}$ & -0.08 & -0.07 & & -0.07 & \\
\hline $\mathrm{C}_{2}$ & 0.22 & 0.22 & & 0.23 & & $\mathrm{C}_{2}$ & 0.22 & 0.22 & & 0.23 & & $\mathrm{C}_{2}$ & 0.22 & 0.25 & & 0.31 & \\
\hline$T_{1}$ & -0.07 & -0.07 & & -0.05 & & $T_{1}$ & -0.06 & -0.06 & & -0.06 & & $T_{1}$ & -0.05 & -0.03 & & -0.01 & \\
\hline $\mathbf{A}_{1}$ & 0.00 & 0.00 & & -0.01 & & $\mathbf{A}_{1}$ & -0.01 & -0.01 & & -0.01 & & $\mathbf{A}_{1}$ & 0.06 & 0.11 & 0.02 & 0.10 & \\
\hline $\mathrm{G}_{2}$ & -0.16 & -0.16 & & -0.15 & & $\mathrm{G}_{2}$ & -0.16 & -0.16 & & -0.14 & & $\mathrm{G}_{2}{ }^{\mathbf{0 x o}}$ & -0.24 & 0.63 & 0.97 & 0.53 & 0.97 \\
\hline $\mathrm{ScA}_{3}$ & 0.04 & 0.08 & 0.03 & 0.07 & 0.03 & $\mathrm{ScA}_{3}$ & 0.03 & 0.07 & 0.03 & 0.07 & 0.02 & $\mathrm{ScA}_{3}$ & 0.03 & 0.06 & 0.01 & 0.06 & 0.03 \\
\hline $\mathrm{G}_{4}$ & -0.15 & 0.72 & 0.96 & 0.62 & 0.96 & $\mathrm{G}_{4}{ }^{\text {oxo }}$ & -0.16 & 0.71 & 0.96 & 0.61 & 0.97 & $\mathrm{G}_{4}$ & -0.14 & -0.14 & & -0.13 & \\
\hline
\end{tabular}


Table 8. Cont.

\begin{tabular}{|c|c|c|c|c|c|c|c|c|c|c|c|c|c|c|c|c|c|}
\hline \multicolumn{18}{|c|}{$d s$-DNA Hirshfeld Charge and Spin Density Population } \\
\hline $\mathrm{G}_{5}$ & -0.11 & -0.08 & 0.01 & -0.09 & 0.01 & $\mathrm{G}_{5}$ & -0.11 & -0.08 & 0.01 & -0.08 & 0.01 & $\mathrm{G}_{5}$ & -0.11 & -0.11 & & -0.11 & \\
\hline $\mathbf{A}_{6}$ & 0.03 & 0.04 & & 0.04 & & $\mathbf{A}_{6}$ & 0.03 & 0.04 & & 0.04 & & $\mathbf{A}_{6}$ & 0.03 & 0.03 & & 0.03 & \\
\hline \multicolumn{6}{|c|}{ RcA-DNA } & \multicolumn{6}{|c|}{ 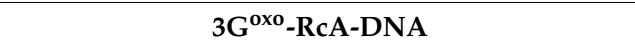 } & \multicolumn{6}{|c|}{ 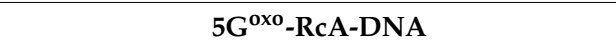 } \\
\hline $\mathrm{T}_{6}$ & 0.05 & -0.05 & & -0.05 & & $\mathrm{~T}_{6}$ & -0.05 & -0.05 & & -0.05 & & $T_{6}$ & -0.05 & -0.05 & & -0.05 & \\
\hline $\mathrm{C}_{5}$ & -0.21 & 0.21 & & 0.22 & & $\mathrm{C}_{5}$ & 0.21 & 0.22 & & 0.23 & & $\mathrm{C}_{5}$ & 0.21 & 0.21 & & 0.21 & \\
\hline $\mathrm{C}_{4}$ & 0.13 & 0.16 & & 0.24 & & $\mathrm{C}_{4}$ & 0.14 & 0.17 & & 0.23 & & $\mathrm{C}_{4}$ & 0.13 & 0.13 & & 0.14 & \\
\hline $\mathbf{T}_{3}$ & -0.06 & -0.04 & & -0.04 & & $T_{3}$ & -0.09 & -0.08 & & -0.08 & & $T_{3}$ & -0.06 & -0.05 & & -0.08 & \\
\hline $\mathrm{C}_{2}$ & 0.22 & 0.23 & & 0.23 & & $\mathrm{C}_{2}$ & 0.21 & 0.21 & & 0.21 & & $\mathrm{C}_{2}$ & 0.23 & 0.26 & & 0.30 & \\
\hline $\mathrm{T}_{1}$ & -0.03 & -0.03 & & -0.03 & & $T_{1}$ & -0.03 & -0.03 & & -0.03 & & $T_{1}$ & -0.04 & -0.02 & & 0.00 & \\
\hline $\mathbf{A}_{1}$ & -0.02 & -0.02 & & -0.02 & & $\mathbf{A}_{1}$ & -0.02 & -0.02 & & -0.02 & & $\mathbf{A}_{1}$ & -0.01 & 0.06 & 0.04 & 0.07 & 0.05 \\
\hline $\mathrm{G}_{2}$ & -0.15 & -0.15 & & -0.14 & & $\mathrm{G}_{2}$ & -0.15 & -0.14 & & -0.13 & & $\mathrm{G}_{2}{ }^{\text {oxo }}$ & -0.17 & 0.66 & 0.95 & 0.55 & 0.92 \\
\hline $\mathrm{RcA}_{3}$ & 0.02 & 0.10 & 0.07 & 0.06 & 0.03 & $\mathrm{RcA}_{3}$ & 0.06 & 0.10 & 0.02 & 0.08 & 0.02 & $\mathrm{RcA}_{3}$ & 0.03 & 0.06 & 0.01 & 0.06 & 0.03 \\
\hline $\mathrm{G}_{4}$ & -0.17 & 0.63 & 0.89 & 0.59 & 0.95 & $\mathrm{G}_{4}{ }^{\text {oxo }}$ & -0.19 & 0.67 & 0.96 & 0.61 & 0.97 & $\mathrm{G}_{4}$ & -0.16 & -0.16 & & -0.13 & \\
\hline $\mathrm{G}_{5}$ & -0.12 & -0.07 & 0.04 & -0.09 & 0.02 & $\mathrm{G}_{5}$ & -0.12 & -0.08 & 0.02 & -0.09 & 0.01 & $\mathrm{G}_{5}$ & -0.12 & -0.12 & & -0.12 & \\
\hline $\mathbf{A}_{6}$ & 0.03 & 0.04 & & 0.04 & & $\mathbf{A}_{6}$ & 0.04 & 0.04 & & 0.05 & & $\mathbf{A}_{6}$ & 0.03 & 0.03 & & 0.04 & \\
\hline
\end{tabular}


Table 9. Energy barriers (in eV) for radical cation migration between base pairs within trimers. The vertical modes, i.e.. the energies of each base pair's radical cation, were calculated for their neutral geometry. The adiabatic modes, i.e.. the energies of each base pair's radical cation, were calculated for their cation geometry. Arrows indicate the direction of the hole transfer from one base pair to another, e.g., $A^{+} \rightarrow G$, calculated at the M062x/6-31+G $G^{* *}$ level of theory in the aqueous phase [42].

\begin{tabular}{|c|c|c|c|c|c|c|c|}
\hline \multirow{3}{*}{$d s$-oligo } & \multirow{3}{*}{ Mode } & \multicolumn{6}{|c|}{ Discussed Trimers } \\
\hline & & \multicolumn{3}{|c|}{$\mathrm{G}_{2} \mathrm{~A}_{3} \mathrm{G}_{4}$} & \multicolumn{3}{|c|}{$\mathrm{A}_{3} \mathrm{G}_{4} \mathrm{G}_{5}$} \\
\hline & & $\mathrm{G}_{2} \rightarrow \mathrm{A}_{3}$ & $A_{3} \rightarrow G_{4}$ & $\mathrm{G}_{2} \rightarrow \mathrm{G}_{4}$ & $A_{3} \rightarrow G_{4}$ & $\mathrm{G}_{4} \rightarrow \mathrm{G}_{5}$ & $A_{3} \rightarrow G_{5}$ \\
\hline \multirow{5}{*}{ N-DNA } & Vertical & 0.49 & -0.49 & -0.03 & -0.49 & 0.73 & -0.04 \\
\hline & Adiabatic & 0.46 & -0.77 & -0.31 & -0.77 & 0.29 & -0.48 \\
\hline & & $\mathrm{G}_{2} \mathrm{~A}_{3}$ & $A_{3} \leftarrow G_{4}$ & $\mathrm{G}_{2} \leftarrow \mathrm{G}_{4}$ & $\mathrm{~A}_{3} \leftarrow \mathrm{G}_{4}$ & $\mathrm{G}_{4} \leftarrow \mathrm{G}_{5}$ & $A_{3} \leftarrow G_{5}$ \\
\hline & Vertical & -0.46 & 1.18 & 0.31 & 1.18 & -0.01 & 0.89 \\
\hline & Adiabatic & -0.46 & 0.77 & 0.31 & 0.77 & -0.29 & 0.48 \\
\hline \multirow{6}{*}{$3 G^{0 \times 0}-N-D N A$} & & $\mathrm{G}_{2} \rightarrow \mathrm{A}_{3}$ & $\mathrm{~A}_{3} \rightarrow{ }^{\mathbf{0 x o}} \mathrm{G}_{4}$ & $\mathrm{G}_{2} \rightarrow{ }^{\mathrm{oxo}} \mathrm{G}_{4}$ & $\mathrm{~A}_{3} \rightarrow{ }^{\text {oxo }} \mathrm{G}_{4}$ & ${ }^{{ }^{\mathrm{xxo}}} \mathrm{G}_{4} \rightarrow \mathrm{G}_{5}$ & $A_{3} \rightarrow G_{5}$ \\
\hline & Vertical & 0.46 & -0.77 & -0.27 & -0.77 & 0.98 & -0.16 \\
\hline & Adiabatic & 0.50 & -1.14 & -0.64 & -1.14 & 0.59 & -0.54 \\
\hline & & $\mathrm{G}_{2} \leftarrow \mathrm{A}_{3}$ & $\mathrm{~A}_{3} \leftarrow^{\mathrm{oxo}} \mathrm{G}_{4}$ & $\mathrm{G}_{2} \leftarrow^{{ }^{\mathrm{oxo}}} \mathrm{G}_{4}$ & $\mathrm{~A}_{3} \leftarrow^{{ }^{\mathrm{oxo}}} \mathrm{G}_{4}$ & ${ }^{{ }^{\circ x o}} G_{4} \leftarrow G_{5}$ & $A_{3} \leftarrow G_{5}$ \\
\hline & Vertical & -0.49 & 1.44 & 0.64 & 1.44 & -0.23 & 0.85 \\
\hline & Adiabatic & -0.50 & 1.14 & 0.64 & 1.14 & -0.59 & 0.54 \\
\hline \multirow{6}{*}{$5 G^{0 \times 0}-N-D N A$} & & ${ }^{\text {oxo }} \mathrm{G}_{2} \rightarrow \mathrm{A}_{3}$ & $A_{3} \rightarrow G_{4}$ & ${ }^{\text {oxo }} \mathrm{G}_{2} \rightarrow \mathrm{G}_{4}$ & $A_{3} \rightarrow G_{4}$ & $\mathrm{G}_{4} \rightarrow \mathrm{G}_{5}$ & $A_{3} \rightarrow G_{5}$ \\
\hline & Vertical & 1.45 & -0.50 & 0.61 & -0.50 & 0.06 & -0.45 \\
\hline & Adiabatic & 1.10 & -0.51 & 0.59 & -0.51 & 0.05 & -0.46 \\
\hline & & ${ }^{\mathrm{oxo}} \mathrm{G}_{2} \leftarrow \mathrm{A}_{3}$ & $A_{3} \leftarrow G_{4}$ & ${ }^{\text {oxo }} \mathrm{G}_{2} \leftarrow \mathrm{G}_{4}$ & $A_{3} \leftarrow G_{4}$ & $\mathrm{G}_{4} \leftarrow \mathrm{G}_{5}$ & $A_{3} \leftarrow G_{5}$ \\
\hline & Vertical & -0.69 & 0.50 & -0.18 & 0.50 & -0.05 & 0.46 \\
\hline & Adiabatic & -1.10 & 0.51 & -0.59 & 0.51 & -0.05 & 0.46 \\
\hline \multirow{6}{*}{ ScA-DNA } & & $\mathrm{G}_{2} \rightarrow \mathrm{ScA}_{3}$ & $\mathrm{ScA}_{3} \rightarrow \mathrm{G}_{4}$ & $\mathrm{G}_{2} \rightarrow \mathrm{G}_{4}$ & $\mathrm{ScA}_{3} \rightarrow \mathrm{G}_{4}$ & $\mathrm{G}_{4} \rightarrow \mathrm{G}_{5}$ & $\mathrm{ScA}_{3} \rightarrow \mathrm{G}_{5}$ \\
\hline & Vertical & 0.55 & -0.51 & 0.02 & -0.51 & 0.71 & -0.11 \\
\hline & Adiabatic & 0.52 & -0.82 & -0.29 & -0.82 & 0.35 & -0.47 \\
\hline & & $\mathrm{G}_{2} \leftarrow \mathrm{ScA}_{3}$ & $\mathrm{ScA}_{3} \leftarrow \mathrm{G}_{4}$ & $\mathrm{G}_{2} \leftarrow \mathrm{G}_{4}$ & $\mathrm{ScA}_{3} \leftarrow \mathrm{G}_{4}$ & $\mathrm{G}_{4} \leftarrow \mathrm{G}_{5}$ & $\mathrm{ScA}_{3} \leftarrow \mathrm{G}_{5}$ \\
\hline & Vertical & -0.46 & 1.16 & 0.35 & 1.16 & -0.05 & 0.82 \\
\hline & Adiabatic & -0.52 & 0.82 & 0.29 & 0.82 & -0.35 & 0.47 \\
\hline
\end{tabular}


Table 9. Cont.

\begin{tabular}{|c|c|c|c|c|c|c|c|}
\hline \multirow{3}{*}{$d s$-oligo } & \multirow{3}{*}{ Mode } & \multicolumn{6}{|c|}{ Discussed Trimers } \\
\hline & & \multicolumn{3}{|c|}{$\mathrm{G}_{2} \mathrm{~A}_{3} \mathrm{G}_{4}$} & \multicolumn{3}{|c|}{$A_{3} G_{4} G_{5}$} \\
\hline & & $\mathrm{G}_{2} \rightarrow \mathrm{A}_{3}$ & $\mathrm{~A}_{3} \rightarrow \mathrm{G}_{4}$ & $\mathrm{G}_{2} \rightarrow \mathrm{G}_{4}$ & $\mathrm{~A}_{3} \rightarrow \mathrm{G}_{4}$ & $\mathrm{G}_{4} \rightarrow \mathrm{G}_{5}$ & $\mathrm{~A}_{3} \rightarrow \mathrm{G}_{5}$ \\
\hline \multirow{6}{*}{ 3G ${ }^{0 \times 0}$-ScA-DNA } & & $\mathrm{G}_{2} \rightarrow \mathrm{ScA}_{3}$ & $\mathrm{ScA}_{3} \rightarrow{ }^{\mathbf{0 x o}} \mathrm{G}_{4}$ & $\mathrm{G}_{2} \rightarrow{ }^{\mathrm{oxo}} \mathrm{G}_{4}$ & $\mathrm{cSA}_{3} \rightarrow{ }^{0 \times 0} \mathrm{G}_{4}$ & ${ }^{{ }^{\text {oxo }}} \mathrm{G}_{4} \rightarrow \mathrm{G}_{5}$ & $\mathrm{ScA}_{3} \rightarrow \mathrm{G}_{5}$ \\
\hline & Vertical & 0.55 & -0.73 & -0.20 & -0.73 & 1.01 & -0.11 \\
\hline & Adiabatic & 0.53 & -1.12 & -0.59 & -1.12 & 0.64 & -0.48 \\
\hline & & $\mathrm{G}_{2} \leftarrow \mathrm{ScA}_{3}$ & $\mathrm{ScA}_{3} \leftarrow^{\mathrm{oxo}} \mathrm{G}_{4}$ & $\mathrm{G}_{2} \leftarrow^{\mathrm{oxo}} \mathrm{G}_{4}$ & $\mathrm{ScA}_{3} \leftarrow^{\mathrm{oxo}} \mathrm{G}_{4}$ & ${ }^{{ }^{\text {oxo }}} \mathrm{G}_{4} \leftarrow \mathrm{G}_{5}$ & $\mathrm{ScA}_{3} \leftarrow \mathrm{G}_{5}$ \\
\hline & Vertical & -0.53 & 1.47 & 0.60 & 1.47 & -0.25 & 0.83 \\
\hline & Adiabatic & -0.53 & 1.12 & 0.59 & 1.12 & -0.64 & 0.48 \\
\hline \multirow{6}{*}{ 5G ${ }^{\text {oxo }}$-ScA-DNA } & & ${ }^{\text {oxo }} \mathrm{G}_{2} \rightarrow \mathrm{ScA}_{3}$ & $\mathrm{ScA}_{3} \rightarrow \mathrm{G}_{4}$ & ${ }^{{ }^{\text {oxo }} \mathrm{G}_{2} \rightarrow \mathrm{G}_{4}}$ & $\mathrm{ScA}_{3} \rightarrow \mathrm{G}_{4}$ & $\mathrm{G}_{4} \rightarrow \mathrm{G}_{5}$ & $\mathrm{ScA}_{3} \rightarrow \mathrm{G}_{5}$ \\
\hline & Vertical & 1.48 & -0.61 & 0.67 & -0.61 & 0.05 & -0.59 \\
\hline & Adiabatic & 1.28 & -0.64 & 0.64 & -0.64 & 0.03 & -0.61 \\
\hline & & ${ }^{{ }^{\text {xo }}} \mathrm{G}_{2} \leftarrow \mathrm{ScA}_{3}$ & $\mathrm{ScA}_{3} \leftarrow \mathrm{G}_{4}$ & ${ }^{{ }^{\text {oxo }}} \mathrm{G}_{2} \leftarrow \mathrm{G}_{4}$ & $\mathrm{ScA}_{3} \leftarrow \mathrm{G}_{4}$ & $\mathrm{G}_{4} \leftarrow \mathrm{G}_{5}$ & $\mathrm{ScA}_{3} \leftarrow \mathrm{G}_{5}$ \\
\hline & Vertical & -0.87 & 0.52 & -0.23 & 0.52 & -0.04 & 0.48 \\
\hline & Adiabatic & -1.28 & 0.64 & -0.64 & 0.64 & -0.03 & 0.61 \\
\hline \multirow{6}{*}{ RcA-DNA } & & $\mathrm{G}_{2} \rightarrow \mathrm{RcA}_{3}$ & $\mathrm{RcA}_{3} \rightarrow \mathrm{G}_{4}$ & $\mathrm{G}_{2} \rightarrow \mathrm{G}_{4}$ & $\mathrm{cA}_{3} \rightarrow \mathrm{G}_{4}$ & $\mathrm{G}_{4} \rightarrow \mathrm{G}_{5}$ & $\mathrm{cA}_{3} \rightarrow \mathrm{G}_{5}$ \\
\hline & Vertical & 0.49 & -0.52 & 0.02 & -0.52 & 0.69 & -0.18 \\
\hline & Adiabatic & 0.55 & -0.88 & -0.33 & -0.88 & 0.38 & -0.50 \\
\hline & & $\mathrm{G}_{2} \leftarrow \mathrm{RcA}_{3}$ & $\mathrm{RcA}_{3} \leftarrow \mathrm{G}_{4}$ & $\mathrm{G}_{2} \leftarrow \mathrm{G}_{4}$ & $\mathrm{RcA}_{3} \leftarrow \mathrm{G}_{4}$ & $\mathrm{G}_{4} \leftarrow \mathrm{G}_{5}$ & $\mathrm{RcA}_{3} \leftarrow \mathrm{G}_{5}$ \\
\hline & Vertical & -0.42 & 1.08 & 0.46 & 1.08 & -0.06 & 0.70 \\
\hline & Adiabatic & -0.55 & 0.88 & 0.33 & 0.88 & -0.38 & 0.50 \\
\hline \multirow{6}{*}{$3 G^{0 \times 0}$-RcA-DNA } & & $\mathrm{G}_{2} \rightarrow \mathrm{RcA}_{3}$ & $\mathrm{cA}_{3} \rightarrow{ }^{\mathbf{0 x o}} \mathrm{G}_{4}$ & $\mathrm{G}_{2} \rightarrow \mathrm{G}_{4}$ & $\mathrm{RcA}_{3} \rightarrow{ }^{\mathrm{oxo}} \mathrm{G}_{4}$ & ${ }^{\text {oxo }} \mathrm{G}_{4} \rightarrow \mathrm{G}_{5}$ & $\mathrm{RcA}_{3} \rightarrow \mathrm{G}_{5}$ \\
\hline & Vertical & 0.45 & -0.69 & -0.19 & -0.69 & 0.96 & -0.16 \\
\hline & Adiabatic & 0.50 & -1.12 & -0.63 & -1.12 & 0.62 & -0.50 \\
\hline & & $\mathrm{G}_{2} \leftarrow \mathrm{RcA}_{3}$ & $\mathrm{RcA}_{3} \leftarrow^{\mathrm{oxo}} \mathrm{G}_{4}$ & $\mathrm{G}_{2} \leftarrow^{\mathrm{oxo}} \mathrm{G}_{4}$ & $\mathrm{RcA}_{3} \leftarrow \mathrm{G}_{4}$ & ${ }^{\text {oxo }} G_{4} \leftarrow G_{5}$ & $\mathrm{RcA}_{3} \leftarrow \mathrm{G}_{5}$ \\
\hline & Vertical & -0.47 & 1.39 & 0.66 & 1.39 & -0.20 & 0.76 \\
\hline & Adiabatic & -0.50 & 1.12 & 0.63 & 1.12 & -0.62 & 0.50 \\
\hline \multirow{6}{*}{ 5G G $^{\text {oxo }}$-RcA-DNA } & & ${ }^{{ }^{\mathbf{x} 0}} \mathrm{G}_{2} \rightarrow \mathrm{RcA}_{3}$ & $\mathrm{RcA}_{3} \rightarrow \mathrm{G}_{4}$ & ${ }^{\text {oxo }} \mathrm{G}_{2} \rightarrow \mathrm{G}_{4}$ & $\mathrm{RcA}_{3} \rightarrow \mathrm{G}_{4}$ & $\mathrm{G}_{4} \rightarrow \mathrm{G}_{5}$ & $\mathrm{RcA}_{3} \rightarrow \mathrm{G}_{5}$ \\
\hline & Vertical & 1.36 & -0.53 & 0.67 & -0.53 & 0.08 & -0.61 \\
\hline & Adiabatic & 1.19 & -0.69 & -0.50 & -0.69 & 0.11 & -0.58 \\
\hline & & ${ }^{{ }^{0 \times 0}} \mathrm{G}_{2} \leftarrow \mathrm{RcA}_{3}$ & $\mathrm{RcA}_{3} \leftarrow \mathrm{G}_{4}$ & ${ }^{\text {oxo }} G_{2} \leftarrow G_{4}$ & $\mathrm{RcA}_{3} \leftarrow \mathrm{G}_{4}$ & $\mathrm{G}_{4} \leftarrow \mathrm{G}_{5}$ & $\mathrm{RcA}_{3} \leftarrow \mathrm{G}_{5}$ \\
\hline & Vertical & -0.80 & 0.47 & -0.11 & 0.47 & 0.01 & 0.36 \\
\hline & Adiabatic & -1.19 & 0.69 & -0.50 & 0.69 & -0.11 & 0.58 \\
\hline
\end{tabular}


Table 10. Nuclear relaxation energy $\lambda[\mathrm{eV}]$ and hole transfer rate constant $k_{\mathrm{HT}}\left[\mathrm{s}^{-1}\right]$, energy barrier $\Delta \mathrm{G}[\mathrm{eV}]$, activation energy $E_{\mathrm{a}}[\mathrm{eV}]$, and electron coupling energies $V_{12}[\mathrm{eV}]$ of hole transfer between base pairs, calculated at the M062x/6-31+G** level of theory in the aqueous phase ([42] and references cited therein).

\begin{tabular}{|c|c|c|c|c|c|c|c|c|c|c|c|c|c|c|c|c|c|}
\hline \multirow{3}{*}{ System } & \multicolumn{17}{|c|}{$d s$-DNA Hole Transfer between Stacked Base Pairs } \\
\hline & $\lambda$ & $\Delta \mathrm{G}$ & $E_{\mathrm{a}}$ & $V_{12}$ & $K_{\mathrm{HT}}$ & & $\lambda$ & $\Delta \mathrm{G}$ & $E_{\mathrm{a}}$ & $V_{12}$ & $K_{\mathrm{HT}}$ & & $\lambda$ & $\Delta \mathrm{G}$ & $E_{\mathrm{a}}$ & $V_{12}$ & $k_{\mathrm{HT}}$ \\
\hline & \multicolumn{5}{|c|}{ N-DNA } & \multicolumn{6}{|c|}{$3 \mathrm{G}^{\mathrm{oxo}}$-N-DNA } & \multicolumn{6}{|c|}{$5 G^{0 \times 0}-N-D N A$} \\
\hline $\mathrm{G}_{2} \leftarrow \mathrm{A}_{3}$ & 0.00 & -0.46 & 18.60 & 0.221 & 0.00 & $\mathrm{G}_{2} \leftarrow \mathrm{A}_{3}$ & 0.01 & -0.50 & 10.09 & 0.220 & 0.00 & ${ }^{\mathbf{0} 0} \mathrm{G}_{2} \leftarrow \mathrm{A}_{3}$ & 0.41 & -1.10 & 0.29 & 0.320 & $3.2 \times 10^{10}$ \\
\hline$A_{3} \rightarrow G_{4}$ & 0.28 & -0.77 & 0.22 & 0.246 & $3.8 \times 10^{11}$ & $A_{3} \rightarrow{ }^{0 \times 0} G_{4}$ & 0.37 & -1.14 & 0.41 & 0.363 & $5.2 \times 10^{8}$ & $\mathrm{~A}_{3} \rightarrow \mathrm{G}_{4}$ & 0.01 & -0.51 & 4.31 & 0.246 & 0.00 \\
\hline \multirow[t]{2}{*}{$\mathrm{G}_{4} \leftarrow \mathrm{G}_{5}$} & 0.44 & -0.29 & 0.01 & 0.051 & $4.0 \times 10^{13}$ & ${ }^{\text {oxo }} \mathrm{G}_{4} \leftarrow \mathrm{G}_{5}$ & 0.38 & -0.59 & 0.03 & 0.113 & $1.1 \times 10^{14}$ & $\mathrm{G}_{4} \leftarrow \mathrm{G}_{5}$ & 0.02 & -0.05 & 0.01 & 0.048 & $1.7 \times 10^{14}$ \\
\hline & \multicolumn{5}{|c|}{ ScA-DNA } & \multicolumn{6}{|c|}{ 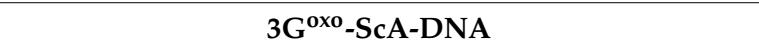 } & \multicolumn{6}{|c|}{$5 \mathrm{G}^{\mathrm{oxo}}$-ScA-DNA } \\
\hline $\mathrm{G}_{2} \leftarrow \mathrm{ScA}_{3}$ & 0.06 & -0.52 & 0.86 & 0.263 & 14.58 & $\mathrm{G}_{2} \leftarrow \mathrm{cSA}_{3}$ & 0.02 & -0.53 & 4.30 & 0.271 & 0.00 & ${ }^{{ }^{x o}} \mathrm{G}_{2} \leftarrow \mathrm{ScA}_{3}$ & 0.41 & -1.28 & 0.45 & 0.367 & $7.3 \times 10^{7}$ \\
\hline $\mathrm{ScA}_{3} \rightarrow \mathrm{G}_{4}$ & 0.31 & -0.82 & 0.21 & 0.264 & $6.4 \times 10^{11}$ & $\mathrm{ScA}_{3} \rightarrow^{\text {oxo }} \mathrm{G}_{4}$ & 0.39 & -1.12 & 0.35 & 0.378 & $5.5 \times 10^{9}$ & $\mathrm{ScA}_{3} \rightarrow \mathrm{G}_{4}$ & 0.04 & -0.64 & 2.62 & 0.271 & 0.00 \\
\hline \multirow[t]{2}{*}{$\mathrm{G}_{4} \leftarrow \mathrm{G}_{5}$} & 0.36 & -0.35 & 0.00 & 0.035 & $3.4 \times 10^{13}$ & ${ }^{{ }^{x o}} \mathrm{G}_{4} \leftarrow \mathrm{G}_{5}$ & 0.37 & -0.64 & 0.05 & 0.157 & $1.0 \times 10^{14}$ & $\mathrm{G}_{4} \leftarrow \mathrm{G}_{5}$ & 0.01 & -0.03 & 0.01 & 0.038 & $1.5 \times 10^{14}$ \\
\hline & \multicolumn{5}{|c|}{ RcA-DNA } & \multicolumn{6}{|c|}{ 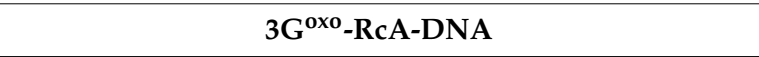 } & \multicolumn{6}{|c|}{ 5Goxo-RcA-DNA } \\
\hline $\mathrm{G}_{2} \leftarrow \mathrm{RcA}_{3}$ & 0.13 & -0.55 & 0.33 & 0.260 & $9.1 \times 10^{9}$ & $\mathrm{G}_{2} \leftarrow \mathrm{RcA}_{3}$ & 0.03 & -0.50 & 1.91 & 0.268 & 0.00 & ${ }^{\mathrm{oxo}} \mathrm{G}_{2} \leftarrow \mathrm{RcA}_{3}$ & 0.39 & -1.19 & 0.41 & 0.352 & $3.28 \times 10^{8}$ \\
\hline $\mathrm{RcA}_{3} \rightarrow \mathrm{G}_{4}$ & 0.35 & -0.88 & 0.19 & 0.297 & $1.3 \times 10^{12}$ & $\mathrm{RcA}_{3} \rightarrow{ }^{\mathbf{0 x 0}} \mathrm{G}_{4}$ & 0.43 & -1.12 & 0.27 & 0.349 & $7.8 \times 10^{10}$ & $\mathrm{RcA}_{3} \rightarrow \mathrm{G}_{4}$ & 0.16 & -0.69 & 0.43 & 0.291 & $1.75 \times 10^{8}$ \\
\hline $\mathrm{G}_{4} \leftarrow \mathrm{G}_{5}$ & 0.31 & -0.38 & 0.00 & 0.086 & $1.9 \times 10^{14}$ & ${ }^{\text {oxo }} G_{4} \leftarrow G_{5}$ & 0.34 & -0.62 & 0.06 & 0.131 & $5.3 \times 10^{13}$ & $\mathrm{G}_{4} \leftarrow \mathrm{G}_{5}$ & 0.12 & -0.11 & 0.00 & 0.085 & $3.5 \times 10^{14}$ \\
\hline
\end{tabular}


The calculated $k_{\mathrm{HT}} \mathrm{S}$ value between the base pair dimers of the reference ds-oligo gives the following values: 0.00 for $\mathbf{G}_{2} \leftarrow \mathrm{A} 3,3.8 \times 10^{11}$ for $\mathbf{A}_{3} \rightarrow \mathrm{G}_{4} 4.0 \times 10^{13}$, and $\mathbf{G}_{4} \leftarrow \mathrm{G}_{5}$. The obtained higher value for $\mathrm{G}_{4} \leftarrow \mathrm{G}_{5}$ is in good agreement with recent theoretical studies, which have postulated that the hole migrated in the $5^{\prime}$-end direction of GG dimers [32]. The single lesion formation in the double helix influence on the $\mathrm{CT}$ process depends on its place of settlement. The presence of $\mathrm{G}^{\text {oxo }}$ as part of the $\mathrm{G}_{4} \mathrm{G}_{5}$ dimer (on its $5^{\prime}$-end) leads to a greater $\mathrm{CT}$ rate increase by one order of magnitude than for N-DNA, with subsequent significant $k_{\mathrm{HT}}$ decreases for $A_{3} \rightarrow G_{4}$ transfer (Table 9). The $G^{\text {oxo }}$ shift to the $G_{2}$ position causes the CT rate to increase between $A_{3} \rightarrow G_{2^{\prime}}$ in comparison to native DNA up to $3.2 \times 10^{10}$. However. the $k_{\mathrm{HT}}$ assigned for $\mathrm{G}_{4} \rightarrow \mathrm{G}_{5}$ transfer was at the same level as observed for $\mathrm{N}-\mathrm{DNA}$ while $\Delta \mathrm{G}$ decreases were noted as well.

The formation of $5^{\prime}, 8$-cyclo-2'-deoxyadenosine in the double helix leads to different results depending on the $\mathrm{C} 5^{\prime}$ chirality. The $\left(5^{\prime} S\right)$-cdA force the same effect as discussed for a native $d s$-oligo (N-DNA), when it has been considered as a tandem lesion. The configuration inversion on the $\mathrm{C5}^{\prime}$ of cdA forces $k_{\mathrm{HT}} \mathrm{s}$ increases in all the discussed CTs $\left(\left(5^{\prime} \mathrm{R}\right) \mathrm{CA}_{3} \rightarrow \mathrm{G}_{2} ;\left(5^{\prime} R\right) \mathrm{CA}_{3} \rightarrow \mathrm{G}_{4} ; \mathrm{G}_{5} \rightarrow \mathrm{G}_{4}\right)$ (Table 9). These observations indicate that the charge transfer within the double helix can be disturbed by structural changes forced by $\left(5^{\prime} R\right)$-cdA (Tables 2-4). Moreover, based on the energy barrier analysis presented in Table 9. the transfer between $\mathrm{G}_{2} \rightarrow \mathrm{G}_{4}$ can take place in the adiabatic mode $(-0.33 \mathrm{eV})$. More details on calculated energy levels can be found in the Supplementary Materials.

The presence of $G^{\text {oxo }}$ and $\left(5^{\prime} R\right)$ - or $\left(5^{\prime} S\right)$-cdA in the same helix turn leads to clustered damage formation. In the case when $G^{\text {oxo }}$ is part of the ${ }^{\text {oxo }} \mathrm{G}_{4} \mathrm{G}_{5}$ dimer (3G $\mathrm{G}^{\text {oxo }}-\mathrm{ScA}-\mathrm{DNA}$ and $3 \mathrm{G}^{\text {oxo }}$-RcA-DNA). the charge transfer is allowed for $\mathrm{cA}_{3} \rightarrow \mathrm{G}_{4}$ oxo and $\mathrm{G}_{5} \rightarrow \mathrm{G}_{4}{ }^{\text {oxo }}$. However, $\left(5^{\prime} S\right)$-cdA increases the hole migration from $\left(5^{\prime} S\right) \mathrm{CA}_{3} \rightarrow \mathrm{G}_{4}$ by one order of magnitude in comparison with N-DNA, while for the opposite diastereomer, this value was two orders of magnitude higher. Subsequently, (5'S)-cdA left $k_{\mathrm{ET}}$ of $\mathrm{G}_{5} \rightarrow \mathrm{G}_{4}$ oxo at the same level as was assigned for native N-DNA for $\left(5^{\prime} R\right)$-cdA; this value was found to be one order of magnitude lower. The above indicates that depending on the $\mathrm{C}^{\prime}{ }^{\prime}$ chirality, cdA can modulate the charge transfer in its $3^{\prime}$ - or $5^{\prime}$-end direction in the case of a clustered DNA lesion. This was confirmed by the results obtained for 5G ${ }^{\text {oxo }}$-ScA-DNA and 5G ${ }^{\text {oxo }}$-RcA-DNA, where G Goxo $^{\text {was }}$ shifted to the $\mathrm{G}_{2}$ position (Figure 2, Table 1). As presented in Table 10. the presence of $\left(5^{\prime} S\right)$-cdA slows down the $\left(5^{\prime} S\right) \mathrm{CA} \rightarrow \mathrm{G}_{2}{ }^{\text {oxo }}$ charge transfer by three orders of magnitude, while $\left(5^{\prime} R\right)$-cdA is only by two in comparison with $5 \mathrm{G}^{\text {oxo }}-\mathrm{N}-\mathrm{DNA}$. Subsequently, both cdA diastereomers left $\mathrm{G}_{5} \rightarrow \mathrm{G}_{4}$ at the same level as forced by dA in suitable single-lesioned DNA. Surprisingly, in the case of 5G ${ }^{\text {oxo }}$-RcA-DNA. the $\left(5^{\prime} R\right) \mathrm{CA}_{3} \rightarrow \mathrm{G}_{4}$ was found to be allowed/possible $-k_{\mathrm{ET}}=1.75 \times 10^{8}$-and was at the same level as that assigned for $\left(5^{\prime} R\right) \mathrm{CA}_{3} \rightarrow \mathrm{G}_{2}{ }^{\text {oxo }}$, which indicates that the $5^{\prime} R$ diastereomer is able to disturb the charge transfer process. These observations are in good agreement with previous theoretical studies in which the directional effect of cdAs was noted [34].

\section{Materials and Methods}

\subsection{Computation Methodology of QM/MM Studies [42,43]}

The geometry optimizations of $d s$-hexamers presented in Table 1 were performed using the QM/MM strategy [35,36]. The structures of the double-stranded oligonucleotides were divided into high- HL (nucleobases, M06-2X/D95*), and low- LL (sugar-phosphate backbone, UFF) levels of calculation using ONIOM in the aqueous phase [55]. The solvent effect was described for an aqueous medium, applying Tomasi's polarized continuum model [56]. The negative charges of each phosphate group were neutralized by the addition of protons. The full structure optimized ds-hexamers were converted to base pairs by sugar-phosphate backbone removal. In the formed base pair systems. the atoms were saturated, if necessary, with hydrogen atoms. The spatial location of the hydrogen atoms added for saturation were optimized at the M06-2X/D95* level of theory in the aqueous phase, with the position of all other atoms frozen. 


\subsection{Computation Methodology of Density Functional Theory (DFT) Study}

All energy calculations were performed in the aqueous phase by the density functional theory (DFT) using the M06-2X functional with a double- $\zeta 6-31+\mathrm{G}^{* *}$ basis set $[57,58]$. The characterization of the transition dipole moment of excited states and the single point calculation at the M06-2X/6-31+G* level of theory were performed using time-dependent DFT (TD-DFT) methodology [59]. For all the optimized structures, a charge and spin analysis was achieved using Hirshfeld methodology at the M06-2X/6-31+G** level [60]. The electron coupling was calculated using generalized Mulliken-Hush methodology [61]. The electronic properties were calculated as previously described [62]. All calculations were performed in the aqueous phase with the Gaussian 09 (revision A.02) software package [63]. The three-dimensional structural analyses of the mentioned ss- and ds-DNAs, based on a standard reference frame, were obtained with by a 3DNA software package using the web-based interface w3DNA (web 3DNA) [64].

\section{Conclusions}

The appearance of different types of single, tandem, or clustered DNA lesions in the oligonucleotide sequence gives rise to various consequences of charge transfer in comparison with native $d s$-oligo (N-DNA). In this article, for the first time, a comparative analysis was made between unmodified $d s$-oligo and one which contains $\mathrm{G}^{\mathrm{oxo}}, \mathrm{cdA}$, or both. Both types of lesions taken into consideration can be formed by hydroxyl radical activity. However. the $\mathrm{dG}$ oxo by $\bullet \mathrm{OH}$ addition to the $\mathrm{C} 8$ moiety of $\mathrm{dG}$ while the $5^{\prime} R$ and $5^{\prime} S$ diastereomers of $5^{\prime}, 8$-cyclo-2'deoxyadenosine can occur in $d s$-DNA as a result of a two-step cyclization reaction induced by hydrogen atom abstraction from the $\mathrm{C}^{\prime}$ position by a hydrogen radical [65]. These unusual tandem lesions can lead to different local spatial geometry changes in the double helix, next to their place of formation [24]. Probably, as a result, $\left(5^{\prime} R\right)$-cdA and $\left(5^{\prime} S\right)$-cdA(S) had a disparate influence on BER enzyme activities, as well as on the electronic properties of the $d s$-DNA part, next to its appearance. The results presented above indicate that dependent on $\mathrm{C} 5^{\prime}$ chirality, cdA can modulate the charge transfer toward its $3^{\prime}$ - or 5'-end direction in the case of a clustered DNA lesion. However, in all the discussed DNA lesions. the appearance of $\mathrm{dG}^{\text {oxo }}$ in the double helix structure constitutes the final destination of radical cation migration.

Supplementary Materials: The following materials can be online: Table S1. The energies (in Hartree) of Neural, Vertical Cation, Adiabatic Cation and Vertical Neutral forms of ideal base pairs, base pairs extracted from 2lsf.pdb [1] and 5iv1.pdb [2] files calculated at the M062x/6-31+G** level of theory in the aqueous phase. Table S2. The energies (in Hartree) of Neural. Vertical Cation. Adiabatic Cation and Vertical Neutral forms of base pairs extracted from $d s$-oligonucleotides calculated at the M062x/6-31+G $\mathrm{G}^{* *}$ level of theory in the aqueous phase. Table S3. The energies (in Hartree) of Neural. Vertical Cation. Adiabatic Cation and Vertical Neutral forms of base pairs dimers extracted from ds-oligonucleotides calculated at the $\mathrm{M} 062 \mathrm{x} / 6-31+\mathrm{G}^{* *}$ level of theory in the aqueous phase. Table S4. Energies (in Hartree) of Neutral. Vertical Cation. Adiabatic Cation and Vertical Neutral forms of $d s$-trimers extracted from $d s$-oligonucleotides calculated at the M062x/6-31+G** level of theory in the aqueous phase. Table S5. The Ground and Excitation state energies and Excitation and HOMO Energies as well as corresponding Dipole Moments (Ground Excitation and Transition) of base pair dimers extracted from $d s$-oligonucleotides. calculated at the M062x/6-31+G** level of theory in the aqueous phase using the DFT or TD-DFT methodology. Eighteen pdb files of discussed $d s$-oligo structures: ScA_DNA_Neutral.pdb, ScA_DNA_Cation.pdb, RcA_DNA_Neutral, RcA_DNA_Cation.pdb, N_DNA_Neutral.pdb, N_DNA_Cation.pdb, 5Goxo_ScA_DNA_Neutral.pdb, 5Goxo_ScA_DNA_Cation.pdb, 5Goxo_RcA_DNA_Neutral.pdb, 5Goxo_N_DNA_Cation.pdb, 3Goxo_RcA_DNA_Neutral.pdb, 5Goxo_RcA_DNA_Cation.pdb, 3Goxo_ScA_DNA_Neutral.pdb, 3Goxo_RcA_DNA_Cation.pdb, 5Goxo_N_DNA_Neutral.pdb, 3Goxo_ScA_DNA_Cation.pdb, 3Goxo_N_DNA_Cation.pdb.

Funding: This study was supported by the Medical University of Lodz (503/3-045-02/503-31-002), National Science Center, Poland (Grant No. 2016/23/B/NZ7/03367) and in part by PL-Grid infrastructure (Prometheus, ACC Cyfronet AGH).

Conflicts of Interest: The author declares no conflict of interest. 


\section{References}

1. Depamphilis, M.L. Review: Nuclear structure and DNA replication. J. Struct. Biol. 2000, 129, $186-197$. [CrossRef] [PubMed]

2. Cooke, M.S.; Evans, M.D.; Dizdaroglu, M.; Lunec, J. Oxidative DNA damage: Mechanisms, mutation, and disease. FASEB J. 2003, 17, 1195-1214. [CrossRef]

3. Sudhir Ambekar, S. DNA: Damage and Repair Mechanisms in Humans. Glob. J. Pharm. Pharm. Sci. $2017,3$. [CrossRef]

4. Aprioku, J.S. Pharmacology of free radicals and the impact of reactive oxygen species on the testis. J. Reprod. Infertil. 2013, 14, 158-172. [PubMed]

5. Valko, M.; Izakovic, M.; Mazur, M.; Rhodes, C.J.; Telser, J. Role of oxygen radicals in DNA damage and cancer incidence. Mol. Cell. Biochem. 2004, 266, 37-56. [CrossRef]

6. Terato, H.; Ide, H. Clustered DNA damage induced by heavy ion particles. Biol. Sci. Sp. Uchū Seibutsu Kagaku 2004, 18, 206-215. [CrossRef]

7. Imoto, S.; Bransfield, L.A.; Croteau, D.L.; Van Houten, B.; Greenberg, M.M. DNA tandem lesion repair by strand displacement synthesis and nucleotide excision repair. Biochemistry 2008, 47, 4306-4316. [CrossRef]

8. Mangal, D.; Vudathala, D.; Park, J.H.; Seon, H.L.; Penning, T.M.; Blair, I.A. Analysis of 7,8-dihydro8-oxo-2'-deoxyguanosine in cellular DNA during oxidative stress. Chem. Res. Toxicol. 2009, 22, 788-797. [CrossRef]

9. Swenberg, J.A.; Lu, K.; Moeller, B.C.; Gao, L.; Upton, P.B.; Nakamura, J.; Starr, T.B. Endogenous versus exogenous DNA adducts: Their role in carcinogenesis, epidemiology, and risk assessment. Toxicol. Sci. 2011, 120, 130-145. [CrossRef]

10. Guerrero, C.R.; Wang, J.; Wang, Y. Induction of 8,5'-Cyclo-2'-deoxyadenosine and 8,5'-Cyclo-2'deoxyguanosine in Isolated DNA by Fenton-Type Reagents. Chem. Res. Toxicol. 2013, 26, 1361-1366. [CrossRef]

11. Chatgilialoglu, C.; Ferreri, C.; Terzidis, M.A. Purine 5',8-cyclonucleoside lesions: Chemistry and biology. Chem. Soc. Rev. 2011, 40, 1368-1382. [CrossRef] [PubMed]

12. Cadet, J.; Di Mascio, P.; Wagner, J.R. Radiation-induced (5'R)-and (5'S)-purine 5',8-cyclo-2'deoxyribonucleosides in human cells: A revisited analysis of HPLC-MS/MS measurements. Free Radic. Res. 2019, 53, 574-577. [CrossRef] [PubMed]

13. Kuraoka, I.; Robins, P.; Masutani, C.; Hanaoka, F.; Gasparutto, D.; Cadet, J.; Wood, R.D.; Lindahl, T. Oxygen free radical damage to DNA: Translesion synthesis by human DNA polymerase $\eta$ and resistance to exonuclease action at cyclopurine deoxynucleoside residues. J. Biol. Chem. 2001, 276, 49283-49288. [CrossRef] [PubMed]

14. You, C.; Dai, X.; Yuan, B.; Wang, J.; Wang, J.; Brooks, P.J.; Niedernhofer, L.J.; Wang, Y. A quantitative assay for assessing the effects of DNA lesions on transcription. Nat. Chem. Biol. 2012, 8, 817-822. [CrossRef]

15. Kuraoka, I.; Bender, C.; Romieu, A.; Cadet, J.; Wood, R.D.; Lindahl, T. Removal of oxygen free-radical-induced $5^{\prime}, 8$-purine cyclodeoxynucleosides from DNA by the nucleotide excision-repair pathway in human cells. Proc. Natl. Acad. Sci. USA 2000, 97, 3832-3837. [CrossRef]

16. Karwowski, B.T. The Influence of ( $\left.5^{\prime} R\right)$ - and $\left(5^{\prime} S\right)-5^{\prime}, 8-C y c l o-2^{\prime}$-Deoxyadenosine on UDG and hAPE1 Activity. Tandem Lesions are the Base Excision Repair System's Nightmare. Cells 2019, 8, 1303. [CrossRef]

17. Sancar, A.; Lindsey-Boltz, L.A.; Ünsal-Kaçmaz, K.; Linn, S. Molecular Mechanisms of Mammalian DNA Repair and the DNA Damage Checkpoints. Annu. Rev. Biochem. 2004, 73, 39-85. [CrossRef]

18. Evans, M.D.; Dizdaroglu, M.; Cooke, M.S. Oxidative DNA Damage and Disease: Induction, Repair and Significance. Mutat. Res. 2004, 567, 1-61. [CrossRef]

19. Kong, Q.; Lin, C.G. Oxidative damage to RNA: Mechanisms, consequences, and diseases. Cell. Mol. Life Sci. 2010, 67, 1817-1829. [CrossRef]

20. Brooks, P.J. The 8,5'-cyclopurine-2'-deoxynucleosides: Candidate neurodegenerative DNA lesions in xeroderma pigmentosum, and unique probes of transcription and nucleotide excision repair. DNA Repair. (Amst) 2008, 7, 1168-1179. [CrossRef]

21. de Souza-Pinto, N.C. Repair of Oxidative DNA Damage. Brenner's Encycl. Genet. Second Ed. 2013, 35, 142-143. [CrossRef] 
22. Fortini, P.; Parlanti, E.; Sidorkina, O.M.; Laval, J.; Dogliotti, E. The type of DNA glycosylase determines the base excision repair pathway in mammalian cells. J. Biol. Chem. 1999, 274, 15230-15236. [CrossRef]

23. Jacobs, A.L.; Schär, P. DNA glycosylases: In DNA repair and beyond. Chromosoma 2012, 121, 1-20. [CrossRef] [PubMed]

24. Karwowski, B.T. The role of ( $\left(5^{\prime} \mathrm{R}\right)$ and $\left(5^{\prime} \mathrm{S}\right) 5^{\prime}, 8$-cyclo-2'-deoxyadenosine in ds-DNA structure. A comparative QM/MM theoretical study. Comput. Theor. Chem. 2013, 1010, 38-44. [CrossRef]

25. Olson, W.K.; Bansal, M.; Burley, S.K.; Dickerson, R.E.; Gerstein, M.; Harvey, S.C.; Heinemann, U.; Lu, X.J.; Neidle, S.; Shakked, Z.; et al. A Standard Reference Frame for the Description of Nucleic Acid Base-pair Geometry. J. Mol. Biol. 2001, 313, 229-237. [CrossRef] [PubMed]

26. Vasita, R.; Katti, D.S. Nanofibers and their applications in tissue engineering. Int. J. Nanomedicine 2006, 1, 15-30. [CrossRef] [PubMed]

27. Schuster, G.B.; Landman, U. The Mechanism of Long-Distance Radical Cation Transport in Duplex DNA: Ion-Gated Hopping of Polaron-Like Distortions. Proc. Natl. Acad. Sci. USA 2012, 139-161. [CrossRef]

28. Genereux, J.C.; Barton, J.K. Mechanisms for DNA charge transport. Chem. Rev. 2010, 110, 1642-1662. [CrossRef]

29. Sontz, P.A.; Mui, T.P.; Fuss, J.O.; Tainer, J.A.; Barton, J.K. DNA charge transport as a first step in coordinating the detection of lesions by repair proteins. Proc. Natl. Acad. Sci. USA 2012, 109, 1-6. [CrossRef]

30. Fromme, J.C.; Banerjee, A.; Huang, S.J.; Verdine, G.L. Structural basis for removal of adenine mispaired with 8-oxoguanine by MutY adenine DNA glycosylase. Nat. Mater. 2004, 427, 652-656. [CrossRef]

31. Merino, E.J.; Boal, A.K.; Barton, J.K. Biological contexts for DNA charge transport chemistry. Curr. Opin. Chem. Biol. 2008, 12, 229-237. [CrossRef] [PubMed]

32. Kumar, A.; Adhikary, A.; Sevilla, M.D.; Close, D.M. One-electron oxidation of ds $\left(5^{\prime}-G G G-3^{\prime}\right)$ and $\mathrm{ds}\left(5^{\prime}-\mathrm{G}(8 \mathrm{OG}) \mathrm{G}-3^{\prime}\right)$ and the nature of hole distribution: A density functional theory (DFT) study. Phys. Chem. Chem. Phys. 2020, 22, 5078-5089. [CrossRef] [PubMed]

33. Diamantis, P.; Tavernelli, I.; Rothlisberger, U. Vertical Ionization Energies and Electron Affinities of Native and Damaged DNA Bases, Nucleotides, and Pairs from Density Functional Theory Calculations: Model Assessment and Implications for DNA Damage Recognition and Repair. J. Chem. Theory Comput. 2019, 15, 2042-2052. [CrossRef] [PubMed]

34. Karwowski, B.T. Clustered DNA Damage: Electronic Properties and Their Influence on Charge Transfer. 7,8-Dihydro-8-Oxo-2'-Deoxyguaosine Versus 5',8-Cyclo-2'-Deoxyadenosines: A Theoretical Approach. Cells 2020, 9, 424. [CrossRef]

35. Mayhall, N.J.; Raghavachari, K. Charge transfer across ONIOM QM:QM boundaries: The impact of model system preparation. J. Chem. Theory Comput. 2010, 6, 3131-3136. [CrossRef] [PubMed]

36. Lin, H.; Truhlar, D.G. QM/MM: What have we learned, where are we, and where do we go from here? Theor. Chem. Acc. 2007, 117, 1-40. [CrossRef]

37. Plumley, J.A.; Dannenberg, J.J. A comparison of the behavior of functional/basis set combinations for hydrogen-bonding in the water dimer with emphasis on basis set superposition error. J. Comput. Chem. 2011, 32, 1519-1527. [CrossRef]

38. Cammi, R.; Corni, S.; Mennucci, B.; Tomasi, J. Electronic excitation energies of molecules in solution: State specific and linear response methods for nonequilibrium continuum solvation models. J. Chem. Phys. 2005, 122. [CrossRef] [PubMed]

39. Jortner, J.; Bixon, M.; Langenbacher, T.; Michel-Beyerle, M.E. Charge transfer and transport in DNA. Proc. Natl. Acad. Sci. USA 1998, 95, 12759-12765. [CrossRef] [PubMed]

40. Albiser, G.; Lamiri, A.; Premilat, S. The A-B transition: Temperature and base composition effects on hydration of DNA. Int. J. Biol. Macromol. 2001, 28, 199-203. [CrossRef]

41. Breslin, D.T.; Schuster, G.B. Anthraquinone photonucleases: Mechanisms for GG-selective and nonselective cleavage of double-stranded DNA. J. Am. Chem. Soc. 1996, 118, 554-558. [CrossRef]

42. Karwowski, B.T. The AT Interstrand Cross-Link: Structure, Electronic Properties, and Influence on Charge Transfer in dsDNA. Mol. Ther. Nucleic Acids 2018, 13, 665-685. [CrossRef]

43. Romieu, A.; Gasparutto, D.; Molko, D.; Cadet, J. Site-Specific Introduction of (5'S)-5',8-Cyclo-2'deoxyadenosine into Oligodeoxyribonucleotides. J. Org. Chem. 1998, 63, 5245-5249. [CrossRef]

44. Nasr, T.; Li, Z.; Nakagawa, O.; Taniguchi, Y.; Ono, S.; Sasaki, S. Selective fluorescence quenching of the 8-oxoG-clamp by 8-oxodeoxyguanosine in ODN. Bioorganic Med. Chem. Lett. 2009, 19, 727-730. [CrossRef] 
45. Zaliznyak, T.; Lukin, M.; De Los Santos, C. Structure and stability of duplex DNA containing (5'S)-5',8-cyclo-2'-deoxyadenosine: An oxidatively generated lesion repaired by NER. Chem. Res. Toxicol. 2012, 25, 2103-2111. [CrossRef] [PubMed]

46. Hoppins, J.J.; Gruber, D.R.; Miears, H.L.; Kiryutin, A.S.; Kasymov, R.D.; Petrova, D.V.; Endutkin, D.V.; Popov, A.V.; Yurkovskaya, A.V.; Fedechkin, S.O.; et al. 8-oxoguanine affects DNA backbone conformation in the EcoRI recognition site and inhibits its cleavage by the enzyme. PLoS ONE 2016, 11, 1-15. [CrossRef] [PubMed]

47. Sevilla, M.D.; Becker, D.; Yan, M.; Summerfield, S.R. Relative abundances of primary ion radicals in $\gamma$-irradiated DNA: Cytosine vs thymine anions and adenine vs guanine cations. J. Phys. Chem. 1991, 95, 3409-3415. [CrossRef]

48. Sugiyama, H.; Saito, I. Theoretical studies of GG-specific photocleavage of DNA via electron transfer: Significant lowering of ionization potential and $5^{\prime}$-localization of HOMO of stacked GG bases in B-form DNA. J. Am. Chem. Soc. 1996, 118, 7063-7068. [CrossRef]

49. Senthilkumar, K.; Grozema, F.C.; Guerra, C.F.; Bickelhaupt, F.M.; Siebbeles, L.D.A. Mapping the Sites for Selective Oxidation of Guanines in DNA. J. Am. Chem. Soc. 2003, 125, 13658-13659. [CrossRef]

50. Voityuk, A.A.; Jortner, J.; Bixon, M.; Rösch, N. Energetics of hole transfer in DNA. Chem. Phys. Lett. 2000, 324, 430-434. [CrossRef]

51. Kanvah, S.; Schuster, G.B. Long-range oxidative damage to DNA: Protection of guanines by a nonspecifically bound disulfide. J. Am. Chem. Soc. 2002, 124, 11286-11287. [CrossRef] [PubMed]

52. Marcus, R.A. Electron transfer reactions in chemistry. Theory and experiment. Rev. Mod. Phys. 1993, 65, 599-610. [CrossRef]

53. Rust, M.; Lappe, J.; Cave, R.J. Multistate effects in calculations of the electronic coupling element for electron transfer using the generalized Mulliken-Hush method. J. Phys. Chem. A 2002, 106, 3930-3940. [CrossRef]

54. Dreuw, A.; Head-Gordon, M. Single-reference ab initio methods for the calculation of excited states of large molecules. Chem. Rev. 2005, 105, 4009-4037. [CrossRef] [PubMed]

55. Chung, L.W.; Sameera, W.M.C.; Ramozzi, R.; Page, A.J.; Hatanaka, M.; Petrova, G.P.; Harris, T.V.; Li, X.; Ke, Z.; Liu, F.; et al. The ONIOM Method and Its Applications. Chem. Rev. 2015, 115, 5678-5796. [CrossRef]

56. Miertus, S.; Tomasi, J. Approximate evaluations of the electrostatic free energy and internal energy changes in solution processes. Chem. Phys. 1982, 65, 239-245. [CrossRef]

57. Zhao, Y.; Pu, J.; Lynch, B.J.; Truhlar, D.G. Tests of second-generation and third-generation density functionals for thermochemical kinetics. Electronic supplementary information (ESI) available: Mean errors for pure and hybrid DFT methods. See http://www.rsc.org/suppdata/cp/b3/b316260e/. Phys. Chem. Chem. Phys. 2004, 6, 673. [CrossRef]

58. Davidson, E.R. Basis Set Selection for Molecular Calculations. Chem. Rev. 1988, 86, 681-696. [CrossRef]

59. Varsano, D.; Felice, R.D.; Marques, M.A.L.; Rubio, A. A TDDFT Study of the Excited States of DNA Bases and Their Assemblies. J. Phys. Chem. B 2006, 110, 7129-7138. [CrossRef]

60. Marenich, A.V.; Jerome, S.V.; Cramer, C.J.; Truhlar, D.G. Charge model 5: An extension of hirshfeld population analysis for the accurate description of molecular interactions in gaseous and condensed phases. J. Chem. Theory Comput. 2012, 8, 527-541. [CrossRef]

61. Cave, R.J.; Newton, M.D. Generalization of the Mulliken-Hush treatment for the calculation of electron transfer matrix elements. Chem. Phys. Lett. 1996, 249, 15-19. [CrossRef]

62. Karwowski, B.T. The influence of the terminal phosphorothioate diester bond on the DNA oxidation process. An experimental and theoretical approach. Molecules 2015, 20, 12400-12411. [CrossRef] [PubMed]

63. Frisch, M.J.; Trucks, G.W.; Schlegel, H.B.; Scuseria, G.E.; Robb, M.A.; Cheeseman, J.R.; Scalmani, G.; Barone, V.; Mennucci, B.; Petersson, G.A.; et al. Gaussian 09, Revision, A.02; Gaussian Inc.: Wallingford, CT, USA, 2009.

64. Zheng, G.; Lu, X.J.; Olson, W.K. Web 3DNA - A web server for the analysis, reconstruction, and visualization of three-dimensional nucleic-acid structures. Nucleic Acids Res. 2009, 37, 240-246. [CrossRef] [PubMed]

65. Karwowski, B.T. Formation of $5^{\prime}, 8$-cyclo-2' -deoxyadenosine in single strand DNA. Theoretical quantum mechanics study. Org. Biomol. Chem. 2010, 8, 1603-1609. [CrossRef]

Sample Availability: Samples of the compounds are not available from the author.

(C) 2020 by the author. Licensee MDPI, Basel, Switzerland. This article is an open access article distributed under the terms and conditions of the Creative Commons Attribution (CC BY) license (http://creativecommons.org/licenses/by/4.0/). 